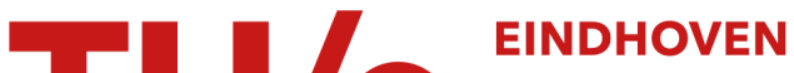 \\ UNIVERSITY OF \\ TECHNOLOGY
}

\section{Single-ion anisotropy of localized-electron compounds}

Citation for published version (APA):

Alders, D., Coehoorn, R., \& Jonge, de, W. J. M. (2001). Single-ion anisotropy of localized-electron compounds. Physical Review B, 63(5), 054407-1/14. [054407]. https://doi.org/10.1103/PhysRevB.63.054407

DOI:

10.1103/PhysRevB.63.054407

Document status and date:

Published: 01/01/2001

\section{Document Version:}

Publisher's PDF, also known as Version of Record (includes final page, issue and volume numbers)

\section{Please check the document version of this publication:}

- A submitted manuscript is the version of the article upon submission and before peer-review. There can be important differences between the submitted version and the official published version of record. People interested in the research are advised to contact the author for the final version of the publication, or visit the $\mathrm{DOI}$ to the publisher's website.

- The final author version and the galley proof are versions of the publication after peer review.

- The final published version features the final layout of the paper including the volume, issue and page numbers.

Link to publication

\section{General rights}

Copyright and moral rights for the publications made accessible in the public portal are retained by the authors and/or other copyright owners and it is a condition of accessing publications that users recognise and abide by the legal requirements associated with these rights.

- Users may download and print one copy of any publication from the public portal for the purpose of private study or research.

- You may not further distribute the material or use it for any profit-making activity or commercial gain

- You may freely distribute the URL identifying the publication in the public portal.

If the publication is distributed under the terms of Article $25 f a$ of the Dutch Copyright Act, indicated by the "Taverne" license above, please follow below link for the End User Agreement:

www.tue.nl/taverne

Take down policy

If you believe that this document breaches copyright please contact us at:

openaccess@tue.nl

providing details and we will investigate your claim. 


\title{
Single-ion anisotropy of localized-electron compounds
}

\author{
D. Alders* \\ Department of Physics, Eindhoven University of Technology, 5600 MB Eindhoven, The Netherlands \\ R. Coehoorn \\ Department of Physics, Eindhoven University of Technology, 5600 MB Eindhoven, The Netherlands \\ and Philips Research Laboratories, Professor Holstlaan 4, 5656 AA Eindhoven, The Netherlands \\ W. J. M. de Jonge \\ Department of Physics, Eindhoven University of Technology, 5600 MB Eindhoven, The Netherlands
}

(Received 6 January 2000; revised manuscript received 5 April 2000; published 3 January 2001)

\begin{abstract}
We present a computational analysis of the influence of taking the full multiplet structure into account in calculations of the single-ion contribution to the magnetocrystalline anisotropy (MCA) of $3 d$ transition-metal (TM) ions in localized-electron compounds at $T=0 \mathrm{~K}$. For atoms with $d^{n}(n=1-4,6-9)$ configurations, at sites with a local cubic, tetragonal, or trigonal symmetry the single ion MCA on the basis of the Hund's rule ground state term only [crystal field (CF) theory] is compared with the single-ion MCA on the basis of a fully relativistic first principles atomic theory including the intra-atomic $d$ - $d$ interaction. Solid state effects are taken into account by effective crystal fields and the exchange field. Under certain, realistic, conditions the use of the full multiplet theory is shown to have a significant effect on the calculated single-ion MCA. We also discuss the effect on the overall cubic anisotropy constants for cubic crystals containing transition metal atom sublattices for which the $d$-metal atoms are located on sites with a local tetragonal and trigonal symmetry. Possible refinements of the theory are discussed.
\end{abstract}

DOI: 10.1103/PhysRevB.63.054407

PACS number(s): 75.30.Gw, 75.50.Gg

\section{INTRODUCTION}

The magnetism of oxidic magnetic materials continues to be an extremely rich field of study. Whereas older work focused on the magnetic properties of hard magnetic hexagonal ferrites, soft magnetic cubic ferrites, and garnets, ${ }^{1}$ recent developments include work on high-temperature superconductor materials containing magnetic ions, perovskites showing the colossal magnetoresistance effect, ${ }^{2}$ and antiferromagnetic oxides showing (in combination with a ferromagnetic thin film) the exchange anisotropy effect. ${ }^{3,4}$ One of the properties of interest is the single-ion contribution to the magnetocrystalline anisotropy (MCA).

This paper is concerned with the calculation of the singleion contribution to the MCA of localized-electron systems such as $3 d$ ions in transition-metal (TM) oxides. This subject has been studied by many authors over the last six decades. We refer to the excellent review article by Darby and Isaac, ${ }^{5}$ and references therein.

The single-ion MCA can be treated theoretically at different levels of rigor. In the phenomenological spinHamiltonian approach ${ }^{6,7}$ the ground state is described in terms of a fictitious spin, whereby the influence of excited states and spin-orbit coupling is simulated by effective parameters. This approach has been used to describe with varying success the observed properties of many systems. ${ }^{5}$ Within this formalism it is possible to formulate analytical expressions for the cubic or axial anisotropy constants. For cubic systems the temperature dependence is shown to be given by the $l(l+1) / 2$ power law, ${ }^{8}$ which relates the anisotropy constants at finite temperature to their value at zero $\mathrm{K}$. In the derivation only two energy levels are considered, which restricts the validity to sufficiently low temperatures. This is in some cases a crude estimation, since the real multiplet may consist of hundreds of closely spaced levels. Moreover, the spin-Hamiltonian treats exchange fields and magnetic fields on equal footing. Single ion anisotropy is, however, closely related with anisotropy of the orbital moment. Therefore the influence of a magnetic field which acts directly on the orbital part is not the same as an exchange field.

Within the framework of crystal field (CF) theory the combined action of electron-correlation, $\mathrm{CF}$ interaction, the exchange field, spin-orbit coupling, and the magnetic field can be described by a single-ion Hamiltonian. In general, all these interaction energies can be of the same order of magnitude, in which case one should solve the full secular equation numerically. However, theoretical discussions presented so far have almost invariably focused on the situation in which all states relevant to the single-ion MCA can be viewed as being derived from the Hund's rule ground state multiplet of the ion considered. A situation in which this approximation apparently fails is when the CF is so large that a high-spin to low-spin transition takes place. The influence of higher multiplets has, as far as we know, only been considered by Baltzer ${ }^{9}$ in a study of the MCA of cubic spinel-type ferrites.

The aim of this paper is to present the results of a computational study of the effect on the single-ion MCA of taking into account the full multiplet structure at $T=0 \mathrm{~K}$. We compare the results obtained from fully relativistic first principles atomic theory including the intra-atomic $d$ - $d$ interaction with the results obtained from the Hund's rule ground term only. We take solid state effects, the exchange field as 
well as spin-orbit interaction into account. The method used has no limitations with respect to the atom, its valence and the symmetry of the ground state. A similar method has been used by Baltzer, ${ }^{9}$ for a specific class of systems. However, he unfortunately made the wrong assumption about the sign of the single-ion MCA constant $K_{1}$ of $\mathrm{Ni}^{2+}$ at the tetrahedral sites of nickel ferrite as compared to what has been found later experimentally and only briefly mentions the very interesting results obtained for $\mathrm{Co}^{2+}$. By means of example we will reconsider in this paper these cases in more detail. The theory as presented can also be extended to rare-earth ions. An example for the case of $4 f$ systems where mixing with excited states becomes important can be found in the breakdown of the linear theory for $\mathrm{Sm}_{2} \mathrm{Fe}_{17} \mathrm{~N}_{3} .{ }^{10}$

In Sec. II we discuss the full single-ion Hamiltonian, which includes the entire multiplet structure. For comparison we introduce in Sec. III a restricted form of the single-ion Hamiltonian, which operates on states within the Hund's rule ground state term only. In Sec. IV the theory of single-ion anisotropy is introduced. The influence of lifting the restriction concerning the basis set used is studied in Sec. V by explicitly comparing the results obtained from the single-ion Hamiltonian as defined in Secs. II and III. We demonstrate that the approximation made by using a restricted basis set may be particularly coarse in some realistic systems in which the ground state becomes heavily mixed. In Sec. VI we reconsider three examples of spinel-type ferrites which have been extensively discussed in the literature, because they involve the magnetically interesting ions $\mathrm{Ni}^{2+}, \mathrm{Fe}^{2+}$, and $\mathrm{Co}^{2+}$ for which good experimental data close to $T=0 \mathrm{~K}$ is available. The influence of mixing in of character of excited states is demonstrated. In Sec. VII we review the implicit assumptions made and discuss possible refinements of the theory. Finally we end with the conclusions in Sec. VIII.

\section{FULL SINGLE-ION HAMILTONIAN}

In the Russell-Saunders scheme, Coulomb and exchange interactions are taken care of by considering electrons coupled together to form a single system characterized by total orbital and total spin quantum number $L$ and $S$. There are several different possible combinations of $L$ and $S$ giving rise to various term symbols ${ }^{2 S+1} L$, where $L$ is denoted in spectroscopic notation as $L=0,1,2,3, \ldots$ for $S, P, D$, $F, \ldots$. For $3 d$ TM systems the term separations are of the order electron volts and thus one is often only interested in the one having the lowest energy. In this weak field approximation Hund's rules can be used to find the free ion ground state term symbol which for TM ions can be described by either $S, D$, or $F .^{11}$ In a solid the ions interact with the CF of the surrounding lattice, which is often viewed as the effect of the electric charge distribution due to ligands on the electronic levels in a crystal, although the aspherical charge distribution of the cation derived $4 p$ orbitals, or the effect of $\mathrm{TM}(3 d)-\mathrm{O}(2 p)$ hybridization can also contribute. In many oxide crystal structures, this field often has predominantly cubic symmetry, with small distortions, in the sense that the splitting of the orbital states due to the cubic field is larger than that due to CF terms of lower symmetry. Let us now

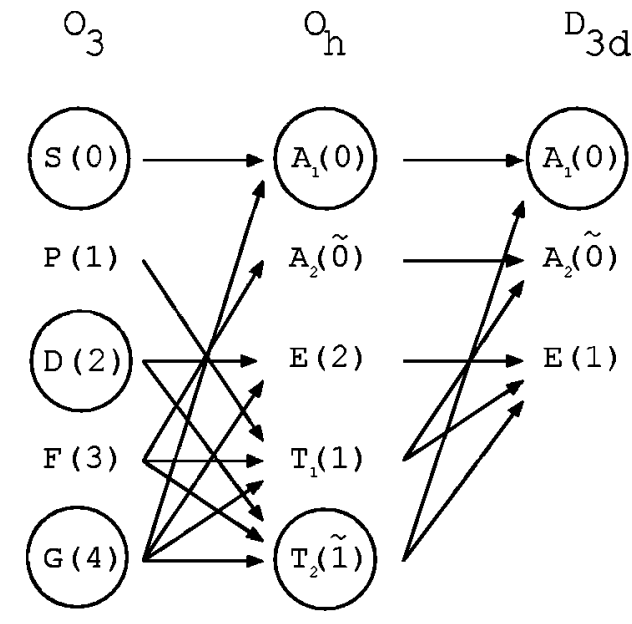

FIG. 1. Branching rules for $O_{3} \rightarrow O_{h} \rightarrow D_{3 d}$ (spherical $\rightarrow$ cubic $\rightarrow$ trigonal symmetry) in Schönflies (Butler) notation. The columns contain all irreducible representations within each symmetry. Encircled are all representations which branch to $A_{1}(0)$ symmetry.

consider the influence of a cubic octahedral or tetrahedral $\mathrm{CF}$. In group theory one can consider a $\mathrm{CF}$ as a reduction from the symmetry group $\mathrm{O}_{3}$ to its subgroup in lower symmetry. All irreducible representations in spherical symmetry are thereby projected onto the subgroup. This transformation is called branching. At a site with cubic $\left(O_{h}\right)$ point symmetry the $D$ state $(L=2)$ branches into two levels which are doubly $(E)$ and triply $(T)$ degenerate while an $F$ state $(L$ $=3$ ) branches into a singlet $(A)$ and two triplets $\left(T_{1}, T_{2}\right)$, see Figs. 1 and 2. The $S$ state is special in the sense that it cannot be influenced since $L=0$.

Except for the configurations $d^{1}$ and $d^{9}$, there are many excited terms. Mixing in of excited states into the ground state may occur via the CF interaction. ${ }^{12}$ It is the objective of this article to study the influence thereof on the single-ion MCA at $T=0 \mathrm{~K}$.

The full Hamiltonian from which we calculate the singleion anisotropy reads

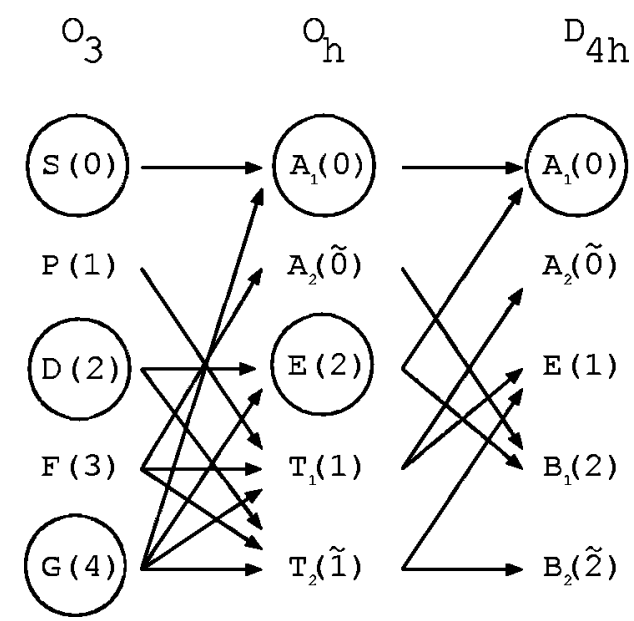

FIG. 2. Branching rules for $O_{3} \rightarrow O_{h} \rightarrow D_{4 h}$ (spherical $\rightarrow$ cubic $\rightarrow$ tetragonal symmetry) in Schönflies (Butler) notation. The columns contain all irreducible representations within each symmetry. Encircled are all representations which branch to $A_{1}(0)$ symmetry. 


$$
\mathcal{H}=\mathcal{H}_{d d}+\mathcal{H}_{\mathrm{CF}}+\mathcal{H}_{\mathrm{ex}}+\mathcal{H}_{\mathrm{SO}}+\mathcal{H}_{\text {Zeeman }},
$$

where $\mathcal{H}_{d d}$ is the term representing the intra-atomic electronelectron Coulomb and exchange interaction, $\mathcal{H}_{\mathrm{CF}}$ is the $\mathrm{CF}$ term, $\mathcal{H}_{\mathrm{ex}}$ is the inter-atomic exchange interaction, $\mathcal{H}_{\mathrm{SO}}$ is the spin-orbit interaction, and $\mathcal{H}_{\text {Zeeman }}$ represents the interaction with the external magnetic field. To solve this Hamiltonian we use the numerical method that has been developed by Cowan $^{13}$ and Butler, ${ }^{14}$ and has been integrated into a single computer program by Thole. ${ }^{15,16}$ The method starts with the calculation of the atomic multiplets (the energy level structure and eigenfunctions for $\mathcal{H}_{\mathrm{CF}}=0$ ). Then the matrix elements for all operators in the spherical group are calculated with the use of Cowan's atomic multiplet program. ${ }^{13}$ Butler's point-group program is then used to obtain the matrix elements in the desired point group. With this general approach one is able to calculate successfully not only the ground state, but also to calculate the transition probabilities between any two configurations in $\mathrm{x}$-ray absorption spectroscopy (XAS), ${ }^{17,18}$ x-ray photoemission spectroscopy (XPS), or Bremsstrahlung isochromat spectroscopy (BIS) in all point group symmetries. ${ }^{19}$

The Slater integrals, which enter the well known expression for the intra-atomic electron-electron Coulomb and exchange term $\mathcal{H}_{d d}$, are reduced to $80 \%$ of their ab initio $\mathrm{HF}$ values (for free space ions) to simulate solid state electron screening effects. This correction method originates from and is supported by the practice of fitting calculated multiplets to optical data. ${ }^{21}$

The crystal field Hamiltonian $\mathcal{H}_{\mathrm{CF}}$ describes the effect of the aspherical electrostatic potential due to the crystal field environment on the electronic levels in a crystal. We note that it is also possible, at least to a finite order of approximation, to consider the $\mathrm{CF}$ Hamiltonian as representing the effect of $\mathrm{TM}(3 d)-\mathrm{O}(2 p)$ hybridization ("ligand field theory"). Most generally, $\mathcal{H}_{\mathrm{CF}}$ may be expressed in terms of an expansion of the $i$ th electron spherical tensors $C_{k q}(i)$ (Ref. 22)

$$
\mathcal{H}_{\mathrm{CF}}=\sum_{k q} B_{k q} \sum_{i=1}^{N} C_{k q}(i),
$$

where the crystal field parameters $B_{k q}$ can be expressed as the product of a factor $A_{k q}$, which depends on the aspherical potential only, and the radial expectation value $\left\langle r^{k}\right\rangle$ of the TM $d$-electron shell: $B_{k q}=A_{k q}\left\langle r^{k}\right\rangle$. In practice $A_{k q}\left\langle r^{k}\right\rangle$ is treated as an inseparable $\mathrm{CF}$ parameter.

For sites with a certain point group symmetry crystal field parameters $A_{k q}$ or $B_{k q}$ with different $(k, q)$ can have a certain, fixed, linear relationship. In subsequent sections we return to this point for the case of $D_{3 d}$ and $D_{4 h}$ point symmetry. It is thus often possible to reduce the number of terms contained in $\mathcal{H}_{\mathrm{CF}}$. Such a more compact form of the $\mathrm{CF}$ Hamiltonian, which is fully adapted to the point symmetry at the site considered, can be obtained by considering the branching rules, such as those given in Figs. 1 and 2 for the $D_{3 d}$ and $D_{4 h}$ point groups, respectively. Apart from defining the splitting of levels upon lowering the point symmetry (as discussed above) these rules can also be used to construct the proper linear contributions of the spherical tensor $C_{k q}$, into unitary tensors $U^{k a 0}$ in which the more compact form of the Hamiltonian is expressed:

$$
\mathcal{H}_{\mathrm{CF}}=\sum_{k a} X^{k a 0} U^{k a 0}
$$

where $X^{k a 0}$ are new crystal field parameters (so-called "Butler notation', ) which are linear combinations of the $B_{k q}$ parameters. The tensor $U$ has dimension energy, while $X$ is dimensionless. The number of relevant unitary operators $U$ is obtained by looking for all branching that produce the $A_{1}(0)$ representation in the point group of consideration. Their form has been given by Racah. ${ }^{23}$ Let us consider as an example three cases of interest: cubic $\left(O_{h}\right)$, trigonal $\left(D_{3 d}\right)$, and tetragonal $\left(D_{4 h}\right)$ point symmetry. It follows from Figs. 1 and 2 that the cubic $\left(O_{h}\right) \mathrm{CF}$ Hamiltonian is given by

$$
\mathcal{H}_{\mathrm{CF}}^{O_{h}}=X^{00} U^{00}+X^{400} U^{400} .
$$

As the first term just produces an energy shift that is identical for all states, we will ignore this term. The trigonal $\left(D_{3 d}\right) \mathrm{CF}$ Hamiltonian is given by

$$
\mathcal{H}_{\mathrm{CF}}^{D_{3 d}}=\mathcal{H}_{\mathrm{CF}}^{O_{h}}+X^{2 \tilde{1} 0} U^{2 \tilde{10}}+X^{4 \tilde{10}} U^{4 \tilde{1} 0} .
$$

Similarly, the tetragonal $\left(D_{4 h}\right) \mathrm{CF}$ Hamiltonian is given by

$$
\mathcal{H}_{\mathrm{CF}}^{D_{4 h}}=\mathcal{H}_{\mathrm{CF}}^{O_{h}}+X^{220} U^{220}+X^{420} U^{420}
$$

A trigonal or a tetragonal distortion of the cubic field thus introduces additional CF parameters. When applying our theory to practical systems we will neglect the parameters of highest order $\left(X^{4 \tilde{10}}=0\right.$ for the trigonal sites, $X^{420}=0$ for tetragonal sites). The full relationship between the $B_{k q}$ and $X^{k a 0} \mathrm{CF}$ parameters can be found in Butler. ${ }^{14}$

The (super) exchange interaction between the TM $d$ electrons on atom $i$ with a neighboring atom $j$ via intervening $\mathrm{O}^{2-}$ ions is described by

$$
\mathcal{H}_{\mathrm{ex}}(i)=g \mu_{0} \mu_{B} \mathbf{H}_{\mathrm{ex}} \cdot \mathbf{S}_{i},
$$

where $\mathbf{H}_{\mathrm{ex}}=\Sigma_{j} J_{i j} \mathbf{S}_{j}$ is the local exchange field, and $J_{i j}$ is the Heisenberg exchange coupling constant, it favors parallel (antiparallel) spins if $J_{i j}<0 \quad\left(J_{i j}>0\right)$. This field is assumed to lie parallel to the direction of magnetization and its magnitude is assumed to be independent of its direction with respect to the crystal axes. We take $g$ equal to its spin only value of 2.0023 .

The spin-orbit Hamiltonian acting on single electron states ( $i$, with $i=1$ to $n$ ) for an ion with configuration $d^{n}$ is

$$
\mathcal{H}_{\mathrm{SO}}=\sum_{i}^{n} \zeta\left(\boldsymbol{l}_{i} \cdot \mathbf{s}_{i}\right),
$$

where $\zeta$ is the single electron spin-orbit parameter. Hartree Fock (HF) values calculated for the $3 d$-TM ions are tabulated in Ref. 20. Spin-orbit interaction in solids is known to be reduced from the free ion values. ${ }^{21}$ However, in this paper we use the $a b$ initio HF spin-orbit values, unless stated otherwise. 
The Hamiltonian that represents the interaction with the external magnetic field $\mathbf{H}$ is given by

$$
\mathcal{H}_{\text {Zeeman }}=\mu_{0} \mu_{B} \mathbf{H}(\mathbf{L}+2 \mathbf{S}) .
$$

In the case of strong magnetic anisotropy the external field interaction energy can become comparable in magnitude to the exchange interaction. A large field is then required to rotate the magnetization, which in some cases can have a nontrivial effect on the electronic level structure and the absolute size of the magnetic moments.

\section{RESTRICTED SINGLE-ION HAMILTONIAN}

Instead of the full Hamiltonian presented in the previous section a restricted single-ion Hamiltonian is now considered, which acts on states within the Hund's rule ground state $(L, S)$ term only:

$$
\mathcal{H}=\mathcal{H}_{\mathrm{CF}}+\mathcal{H}_{\mathrm{ex}}+\mathcal{H}_{\mathrm{SO}}+\mathcal{H}_{\text {Zeeman }},
$$

where the various terms have the meaning already discussed in the previous section. Obviously, the interatomic Coulomb and exchange interaction $\mathcal{H}_{d d}$ is not taken into account explicitly. Implicitly its effect is of course taken into account in part in as far it determines the Hund's rule ground term. For ground terms obeying Hund's rules one must use a different expression, with respect to the previous section, for the spinorbit interaction, viz., $\mathcal{H}_{\mathrm{SO}}=\lambda \overrightarrow{\mathbf{L}} \cdot \overrightarrow{\mathbf{S}}$. The parameters $\zeta$ and $\lambda$ are related by $\lambda= \pm(\zeta / 2 S)$ where the plus sign is required for a shell less than half filled and the minus sign for a shell that is more than half filled. Frequently, the CF Hamiltonian is expressed in terms of the so-called extended Stevens operators $O_{k}^{q}$, and is given by

$$
\mathcal{H}_{\mathrm{CF}}=\sum_{k=2,4,6} \sum_{q=-k}^{+k} A_{k}^{q}\left\langle r^{n}\right\rangle \theta_{n} O_{k}^{q} \equiv \sum_{k=2,4,6} \sum_{q=-k}^{+k} B_{k}^{q} O_{k}^{q},
$$

with the CF parameters $A_{k}^{q}$, the expectation values of $\left\langle r^{n}\right\rangle$ of the radial part of the wave function, the Stevens coefficients $\theta_{n}$, the Stevens operators $O_{k}^{q}$, and finally the CF parameters $B_{k}^{q}$ which are equal to $A_{k}^{q}\left\langle r^{n}\right\rangle \theta_{n}$. We refer to the original paper by Stevens ${ }^{25}$ for an introduction to this method. Various authors have introduced modified conventions and notations. In Eq. (11) we have used the extended Stevens operators $O_{k}^{q},-k \leqslant q \leqslant k$. These are related to the operators $O_{l}^{ \pm m}$ introduced by Buckmaster ${ }^{27}$ by the expressions

$$
\begin{gathered}
O_{k}^{0}=O_{l}^{0}, \\
O_{k}^{q}=\frac{1}{2}\left(O_{l}^{+m}+O_{l}^{-m}\right), \quad m, q>0, \\
O_{k}^{q}=\frac{1}{2 i}\left(O_{l}^{+m}-O_{l}^{-m}\right), \quad m>0, q<0
\end{gathered}
$$

with $l=k$ and $m=|q|$. The operators $O_{l}^{ \pm m}$ are related by rational factors to another set of tensor operators namely $\widetilde{O}_{l m} \cdot{ }^{27}$ In Ref. 28 these operators $\widetilde{O}_{l m}$ are called $O_{q}^{(k)}$ and are
TABLE I. Relationship between some CF parameters as defined using the notation of Butler and the $B_{k}^{q}$ crystal field parameters. The parameter $B_{4}$ is defined in Sec. III.

\begin{tabular}{lcc}
\hline \hline $\begin{array}{l}\text { Point } \\
\text { symmetry }\end{array}$ & $L=2$ & $L=3$ \\
\hline$O_{h}$ & $X^{400}=72 \sqrt{30} B_{4}$ & $X^{400}=360 \sqrt{66} B_{4}$ \\
$D_{4 h}$ & $X^{220}=-3 \sqrt{70} B_{2}^{0}$ & $X^{220}=-6 \sqrt{105} B_{2}^{0}$ \\
$D_{3 d}$ & $X^{2 \tilde{1} 0}=-3 \sqrt{70} B_{2}^{0}$ & $X^{2 \tilde{1}}=-6 \sqrt{105} B_{2}^{0}$ \\
\hline \hline
\end{tabular}

listed for $k=0$ to 6 , for all $q$ values. From the combined Refs. 27 and 28 one may thus obtain explicit forms of the extended Stevens operators $O_{k}^{q}$. A general analytical expression is given for the matrix elements $\left\langle j, m^{\prime}\left|O_{q}^{(k)}\right| j, m\right\rangle$ in Ref. 28 . This is particularly suited for machine computation. The matrix elements have been tabulated by Birgeneau. ${ }^{29}$ One should note that the CF Hamiltonian used [Eq. (11)] is valid in the restricted subspace of a given $L$ or $J$ multiplet. Strictly speaking, it is therefore inappropriate to refer to the $A_{k}^{q}$ or $B_{k}^{q}$ parameters as CF parameters. ${ }^{7}$ The $A_{k}^{q}$ and $B_{k}^{q}$ parameters should not be confused with the real CF parameters $A_{k q}$ and $B_{k q}$ as defined in the previous section. We therefore choose to work with the $X$ parameters as defined in Eq. (3).

In a cubic $\left(O_{h}\right.$ or $\left.T_{d}\right)$ field the restricted CF Hamiltonian contains for $d$ states only two relevant terms

$$
\mathcal{H}_{\mathrm{CF}}^{O_{h}}=B_{4}\left(O_{4}^{0}+5 O_{4}^{4}\right),
$$

where $B_{4}=B_{4}^{0}=\frac{1}{5} B_{4}^{4}$. In a tetragonal $\left(D_{4 h}\right)$ field the restricted $\mathrm{CF}$ Hamiltonian is given by

$$
\mathcal{H}_{\mathrm{CF}}^{D_{4 h}}=B_{4}\left(O_{4}^{0}+5 O_{4}^{4}\right)+B_{2}^{0} O_{2}^{0}+B_{4}^{0} O_{4}^{0},
$$

where the magnitude of the cubic $\mathrm{CF}$ is denoted by $B_{4}$ which is not equal to $B_{4}^{0}$ in this case and the other two terms represent the tetragonal distortion of second and fourth degree in the potential, respectively. The $\hat{z}$ axis is a fourfold axis. Finally, in a trigonal $\left(D_{3 d}\right)$ field the CF Hamiltonian is given by

$$
\mathcal{H}_{\mathrm{CF}}^{D_{3 d}}=-\frac{2}{3} B_{4}\left(O_{4}^{0}+20 \sqrt{2} O_{4}^{3}\right)+B_{2}^{0} O_{2}^{0}+B_{4}^{0} O_{4}^{0},
$$

where the introduction of the factor $-\frac{2}{3}$ makes the parameter $B_{4}$ of the CF operator identical with that in Eq. (13). The $\hat{z}$ axis is a threefold axis. The relationship between the $B_{k}^{q}$ crystal field parameters and the CF parameters ( $X$ parameters) as defined in Eq. (3) are tabulated in Table I.

Transformation matrices for a general rotation $(\theta, \phi)$ of the frame of coordinates of the Stevens operators $O_{k}^{q}$ are given by ${ }^{26}$

$$
\left\{\mathbf{O}_{k}\right\} \equiv \mathbf{S}_{k} \cdot\left[\mathbf{O}_{k}\right],
$$

where $\mathbf{S}_{k}(\theta, \phi)$ is the transformation matrix for the Stevens operators $O_{k}^{q}$ for a given $k$. The curly brackets denote the operators $O_{k}^{q}$ in the original axis system, whereas the square brackets denote the operators $O_{k}^{q}$ in the transformed axis sys- 
tem which is rotated by an azimuthal angle $\phi$ about the original $z$ axis and a polar angle $\theta$ about the new $y$ axis. These transformation matrices are used to rotate the $\mathrm{CF}$ in the desired orientation while, by definition, the exchange and/or magnetic field remains along the $\hat{z}$ direction. This is relevant when calculating the MCA, i.e., the ground state energy as a function of the magnetization direction with respect to the crystal axes.

\section{SINGLE-ION ANISOTROPY}

Let us consider the free energy of an isolated ion with $L$ $\neq 0$. Spin-orbit coupling combined with an arbitrarily small external or exchange field, which defines the quantization axis, already leads to the formation of an aspherical charge cloud (at $T=0 \mathrm{~K}$ ). Upon embedding the ion into a crystal structure one may find extrema in the free energy of the ion as a function of the angle between the direction of the exchange field and/or magnetic field and the crystal axes, due to the electrostatic interaction between the aspherical $3 d$ charge cloud and the aspherical electrostatic potential at the ionic site. This single-ion contribution results from the spinorbit interaction, which couples the spin moment to the orbital moment, and hence to certain preferred crystal directions. In the limit of a very small CF there is a direct linear relation between the free energy and the CF. This leads to an initial linear increase of the anisotropy constants with the $\mathrm{CF}$ parameter. These constants are then determined by the product of the asphericity of the charge cloud in the zero CF limit (the Stevens coefficient $\theta_{n}$, see Sec. III) times the relevant $(k, q)$ component of the aspherical electrostatic potential, as is almost always the case for rare earth materials. This situation changes when the orbital moment becomes more an more quenched by the $\mathrm{CF}$. As a result the spin and the orbital moment become less rigidly coupled, and the anisotropy constants start to decrease with increasing CF. In the limit of an infinite $\mathrm{CF}$ the orbital moment vanishes and the anisotropy constants go to zero.

The free energy $\mathcal{F}\left(\alpha_{1}, \alpha_{2}, \alpha_{3}\right)$ of a magnetically saturated, single domain crystal may be formally expressed as a series expansion in ascending powers of the direction cosines $\alpha_{i}$ of the magnetization with respect to a set of rectangular Cartesian coordinate axes as

$$
\mathcal{F}\left(\alpha_{1}, \alpha_{2}, \alpha_{3}\right)=\overleftrightarrow{b}_{i} \alpha_{i}+\overleftrightarrow{b}_{i j} \alpha_{i} \alpha_{j}+\overleftrightarrow{b}_{i j k} \alpha_{i} \alpha_{j} \alpha_{k}+\cdots
$$

In this equation $\overleftrightarrow{b}_{i}, \overleftrightarrow{b}_{i j}, \ldots$, are components of first, second, and higher rank tensors, of which the properties depend on the crystallographic symmetry. Tensors up to rank 6 have been derived for all crystallographic groups by Fieschi and Fumi. ${ }^{30}$ For cubic crystals the free energy is more compactly expressed by ${ }^{31}$

$$
\begin{aligned}
\mathcal{F}\left(\alpha_{1}, \alpha_{2}, \alpha_{3}\right)= & K_{0}+K_{1} s+K_{2} p+K_{3} s^{2}+K_{4} s p+K_{5} s^{3} \\
& +K_{6} p^{2}+\cdots
\end{aligned}
$$

where $s \equiv \alpha_{1}^{2} \alpha_{2}^{2}+\alpha_{1}^{2} \alpha_{3}^{2}+\alpha_{2}^{2} \alpha_{3}^{2}$ and $p \equiv \alpha_{1}^{2} \alpha_{2}^{2} \alpha_{3}^{2} . K_{1}, K_{2}$, etc., are called the first, second, and higher anisotropy constants.

For crystals of tetragonal or trigonal symmetry the free energy can more compactly be expressed as a function of the polar and azimuthal angle $\theta$ and $\phi$, respectively, when $\theta$ is the angle of the magnetization with respect to the tetragonal or trigonal axis. ${ }^{32}$ For crystals of trigonal symmetry, the free energy can be expressed as

$$
\begin{aligned}
\mathcal{F}(\theta, \phi)= & \widetilde{K}_{0}+\widetilde{K}_{1} \sin ^{2} \theta+\widetilde{K}_{2} \sin ^{4} \theta \\
& +\widetilde{K}_{3} \sin ^{3} \theta \cos \theta \cos (3 \phi)+\widetilde{K}_{4} \sin ^{6} \theta \cos (6 \phi) \\
& +\widetilde{K}_{5} \sin ^{6} \theta+\widetilde{K}_{6} \sin ^{3} \theta \cos ^{3} \theta \cos ^{3}(3 \phi)+\cdots
\end{aligned}
$$

For crystals of tetragonal symmetry, the free energy can be expressed as

$$
\begin{aligned}
\mathcal{F}(\theta, \phi)= & \bar{K}_{0}+\bar{K}_{1} \sin ^{2} \theta+\bar{K}_{2} \sin ^{4} \theta+\bar{K}_{3} \sin ^{4} \theta \sin ^{2} \phi \cos ^{2} \phi \\
& +\cdots
\end{aligned}
$$

Crystals may have an overall symmetry which is higher than the point symmetry of the sites at which the cations reside that contribute to the single ion anisotropy. In cubic crystals with cations at sites with trigonal or tetragonal point symmetry, the local symmetry is described by Eqs. (19) and (20), respectively. The anisotropy constants defined with respect to the overall symmetry, hereafter referred to as global symmetry, may then be obtained via two independent methods. First, one may perform a calculation on the basis of the "local" free energy surface of the local anisotropy constants $K_{i}^{l}$, where $l$ is short for local, and obtain the global anisotropy constants $K_{i}^{g}$, where $g$ is short for global, by making use of general expressions which relate both sets of anisotropy constants. Although formally correct, this method often has the following practical disadvantage. For realistic systems (i.e., when using realistic CF parameters) the local anisotropy constants $K_{i}^{l}$ can in some cases be very small. The introduction of small errors in the determination of local anisotropy constants may subsequently result in large errors in the global values. An accuracy far better than $1 \times 10^{-7} \mathrm{eV}$ for the eigenvalues of the ground state orbitals is sometimes required, which is outside the present accuracy of our full multiplet program. Second, in case one is only interested in the overall global anisotropy constants an alternative method is to perform a direct calculation of the free energy surface $\mathcal{F}\left(\alpha_{1}, \alpha_{2}, \alpha_{3}\right)$ in global coordinates, by summing over the contribution of all sites to $\mathcal{F}\left(\alpha_{1}, \alpha_{2}, \alpha_{3}\right)$ for a specific direction $\left[\alpha_{1}, \alpha_{2}, \alpha_{3}\right]$. We will use the latter method in the following.

In this paper we will restrict ourselves to calculate the global cubic single ion MCA constants for systems with local cubic, tetragonal and trigonal point symmetry. An example for the latter may be found in spinel ferrites where numerous measurements of the single MCA constants have been reported. ${ }^{33-35}$ The octahedral site in spinel ferrites is 
TABLE II. Coefficients $a_{i, j}$ entering the relationship $K_{i}^{g}$ $=\Sigma_{j} a_{i, j} K_{j}^{l}$ between the local $K_{j}^{l}$ [Eq. (19)] and the global $K_{i}^{g}$ [Eq. (18)] anisotropy constants, for a cubic lattice containing ions on crystallographically equivalent sites with trigonal point symmetry, with the trigonal axis along the $\langle 111\rangle$ directions.

\begin{tabular}{cccccccc}
\hline \hline & $K_{0}^{l}$ & $K_{1}^{l}$ & $K_{2}^{l}$ & $K_{3}^{l}$ & $K_{4}^{l}$ & $K_{5}^{l}$ & $K_{6}^{l}$ \\
\hline$K_{0}^{g}$ & 4 & $\frac{2}{3}$ & $\frac{4}{9}$ & $\frac{2 \sqrt{2}}{9}$ & $\frac{8}{27}$ & $\frac{8}{27}$ & $\frac{2 \sqrt{2}}{27}$ \\
$K_{1}^{g}$ & 0 & 0 & $\frac{4}{9}$ & $\frac{-10 \sqrt{2}}{9}$ & $\frac{-28}{9}$ & $\frac{8}{9}$ & $\frac{-4 \sqrt{2}}{9}$ \\
$K_{2}^{g}$ & 0 & 0 & 0 & 0 & $\frac{308}{9}$ & $\frac{-16}{9}$ & $\frac{14 \sqrt{2}}{9}$ \\
\hline \hline
\end{tabular}

surrounded by six neighboring oxygen ions, which give rise to a crystal field with a dominant cubic term and a smaller trigonal contribution. The crystal field has trigonal symmetry around one of the $\langle 111\rangle$ directions. The overall symmetry of a spinel crystal is, however, cubic. The relationship between the local trigonal (tetragonal) and global cubic anisotropy constants can be found in Table II (III).

The phenomenological expressions (18), (19), and (20) for the free energy enable one to obtain the anisotropy constants from a calculated free energy surface with a sufficiently dense mesh. Note that the expansions used do not form a orthogonal set, in contrast to the expansion in Eq. (17). Therefore all anisotropy constants change by including higher order terms. It is therefore rather difficult to make a comparison with experimental single-ion MCA constants given in the literature if it is not clearly stated how the value(s) has (have) been obtained. We illustrate this with the following example. In case of a cubic crystal, and assuming that $\mathcal{F}\left(\alpha_{1}, \alpha_{2}, \alpha_{3}\right)$ is fully described by Eq. (18) using terms up to second order, $K_{1}^{g}$ and $K_{2}^{g}$, can be obtained from a calculation (or measurement) of the free energy for the three principle directions:

$$
\begin{aligned}
& K_{1}^{g}=4[\mathcal{F}(1 / \sqrt{2}, 1 / \sqrt{2}, 0)-\mathcal{F}(1,0,0)], \\
K_{2}^{g}= & 27 \mathcal{F}(1 / \sqrt{3}, 1 / \sqrt{3}, 1 / \sqrt{3})-36 \mathcal{F}(1 / \sqrt{2}, 1 / \sqrt{2}, 0) \\
+ & 9 \mathcal{F}(1,0,0),
\end{aligned}
$$

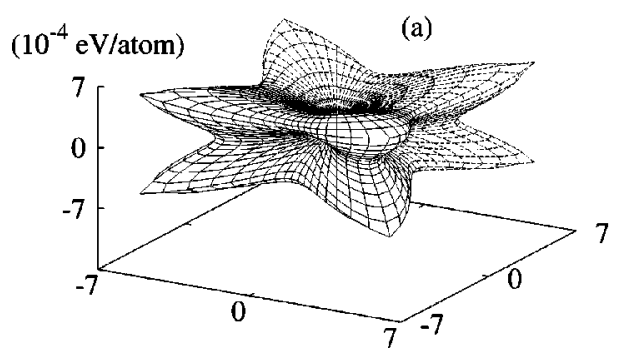

TABLE III. Coefficients $a_{i, j}$ entering the relationship $K_{i}^{g}$ $=\Sigma_{j} a_{i, j} K_{j}^{l}$ between the local $K_{j}^{l}$ [Eq. (20)] and the global $K_{i}^{g}$ [Eq. (18)] anisotropy constants, for a cubic lattice containing ions on crystallographically equivalent sites with tetragonal point symmetry, with the tetragonal axis along the $\langle 100\rangle$ directions.

\begin{tabular}{cccc}
\hline \hline & $K_{1}^{l}$ & $K_{2}^{l}$ & $K_{3}^{l}$ \\
\hline$K_{0}^{g}$ & $\frac{2}{3}$ & $\frac{2}{3}$ & 0 \\
$K_{1}^{g}$ & 0 & $-\frac{2}{3}$ & $\frac{1}{3}$ \\
\hline \hline
\end{tabular}

where $\mathcal{F}\left(\alpha_{1}, \alpha_{2}, \alpha_{3}\right)$ is the free energy of an ion with the exchange and/or magnetic field in the direction $\left[\alpha_{1}, \alpha_{2}, \alpha_{3}\right]$, in global coordinates.

In Fig. 3(a) we show the calculated angular dependence of the free energy with respect to the direction of the exchange field for the $\mathrm{Fe}^{2+}$ ion (using the restricted Hamiltonian [Eq. (10)] with $X^{400}=-18 / \sqrt{30}$ and $\mu_{0} \mu_{B} H_{\mathrm{ex}}=50 \mathrm{meV}$ ). A value of the cubic crystal field parameter of $X^{400}$ $=-18 / \sqrt{30}$ corresponds, by definition, to an energy splitting between $t_{2 g}$ and $e_{g}$ orbitals of $\Delta=-1.0 \mathrm{eV}$. Calculation of $K_{1}$ and $K_{2}$ using Eq. (21) leads to $K_{1}=2.0 \mathrm{meV}$ and $K_{2}$ $=9.0 \mathrm{meV}$. However, it is clear from this example, see Fig. 3(b), that a description in terms of $K_{1}$ and $K_{2}$ only is not always sufficient. A $\chi^{2}$ fit of the full free energy surface using $K_{1}, K_{2}$, and $K_{3}$ leads to $K_{1}=0.3 \mathrm{meV}, K_{2}=3.9 \mathrm{meV}$ (and $K_{3}=6.7 \mathrm{meV}$ ). This clearly shows that the $K$ values obtained can depend significantly on the method used to analyze the calculated results (in experimental data). Unless specified otherwise we will use Eq. (21) to calculate $K_{1}$ and $K_{2}$ in this paper. We notice that in calculational and experimental studies performed so far it is almost never checked how well a restricted set of CF parameters describes the full free energy surface.

We finally note that the phenomenological description of the magnetocrystalline anisotropy in terms of free energy expansions has proven to be quite successful in practice. There are, however, exceptions to this rule. Anomalous behavior of the magnetocrystalline anisotropy can be found in cases when a change in the magnetization direction causes a passage of a sub-band through the Fermi energy in metals. This effect has been observed in $\mathrm{Ni}$ metal at $4 \mathrm{~K}$ by Gersdorf. ${ }^{36}$ In localized electron systems this could happen for systems which are close to a crossing of the ground state

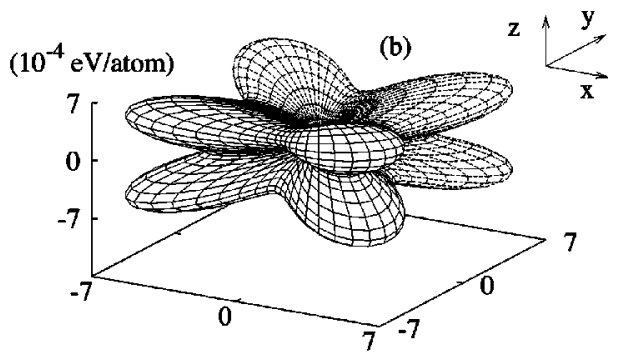

FIG. 3. (a) Angular dependence of the free energy with respect to the direction of the exchange field calculated on the basis of the restricted Hamiltonian for $\mathrm{Fe}^{2+} ; X^{400}=-18 / \sqrt{30}$ (dimensionless parameter, corresponding to $\Delta=-1.0 \mathrm{eV}$ ) and $\mu_{0} \mu_{B} H_{\mathrm{ex}}=50 \mathrm{meV}$. (b) The free energy surface as described by $K_{1}=2.0 \mathrm{meV}$ and $K_{2}=9.0 \mathrm{meV}$. Note that for a more accurate description of the free energy surface given in (a) one needs higher order $K_{i}$ values, see text. 


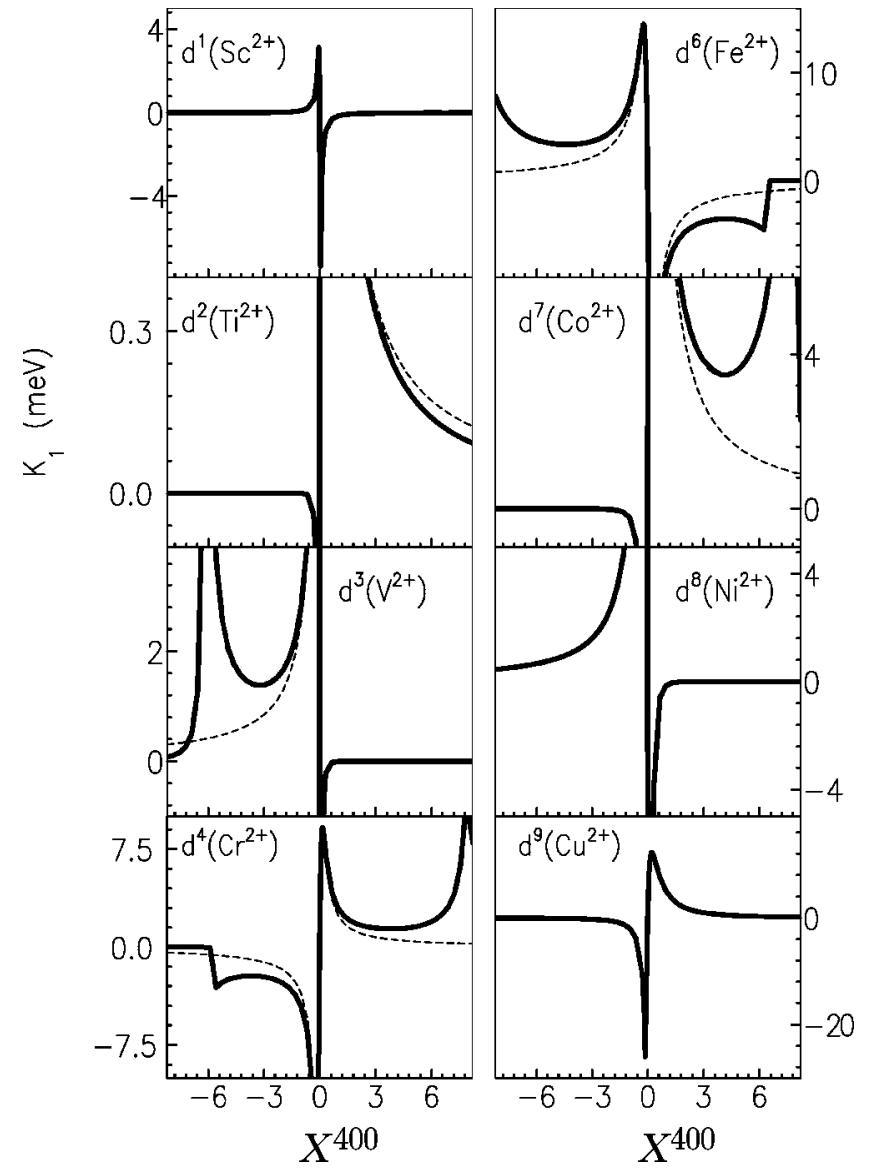

FIG. 4. Calculated cubic single-ion anisotropy constant $K_{1}^{g}$ as a function of the cubic CF parameter $X^{400}$ and a constant exchange field of $\mu_{0} \mu_{B} H_{\mathrm{ex}}=50 \mathrm{meV}$ at $\mathrm{T}=0 \mathrm{~K}$. Full lines give the result from the complete CF theory, in which, as described in Sec. II the basis set includes all terms. Dashed lines represent the result of a basis set that include only the Hund's rule $(L, S, J)$ term. These calculations have been carried out within the CF approach of Sec. III, with $A_{4}^{0}=X^{400} / 72 \sqrt{30} \theta_{4}$.

and first excited state energy levels. A high-spin to low-spin transition induced by a rotation of the spin direction, using an applied magnetic field, would be the most dramatic case. The calculational method presented in this paper is particularly suited for describing such cases, whereas calculations based on the restricted Hamiltonian (which operates on the Hund's rule ground state term only) would obviously fail to describe such cases.

\section{RESULTS: OVERVIEW}

\section{A. Cubic symmetry}

Figures 4 and 5 give an overview of calculated single-ion MCA constants $K_{1}$ and $K_{2}$ for all $3 d^{n}(n=1-4,6-9)$ configurations in cubic symmetry at $T=0 \mathrm{~K}$, as a function of the cubic CF parameter $X^{400}$. The calculations have been carried out using the methods presented in Secs. II and III, with an exchange field corresponding to $\mu_{0} \mu_{B} H_{\mathrm{ex}}=50 \mathrm{meV}$ for zero external magnetic field. We would like to stress that when applying the theory to specific cases it is necessary to esti-
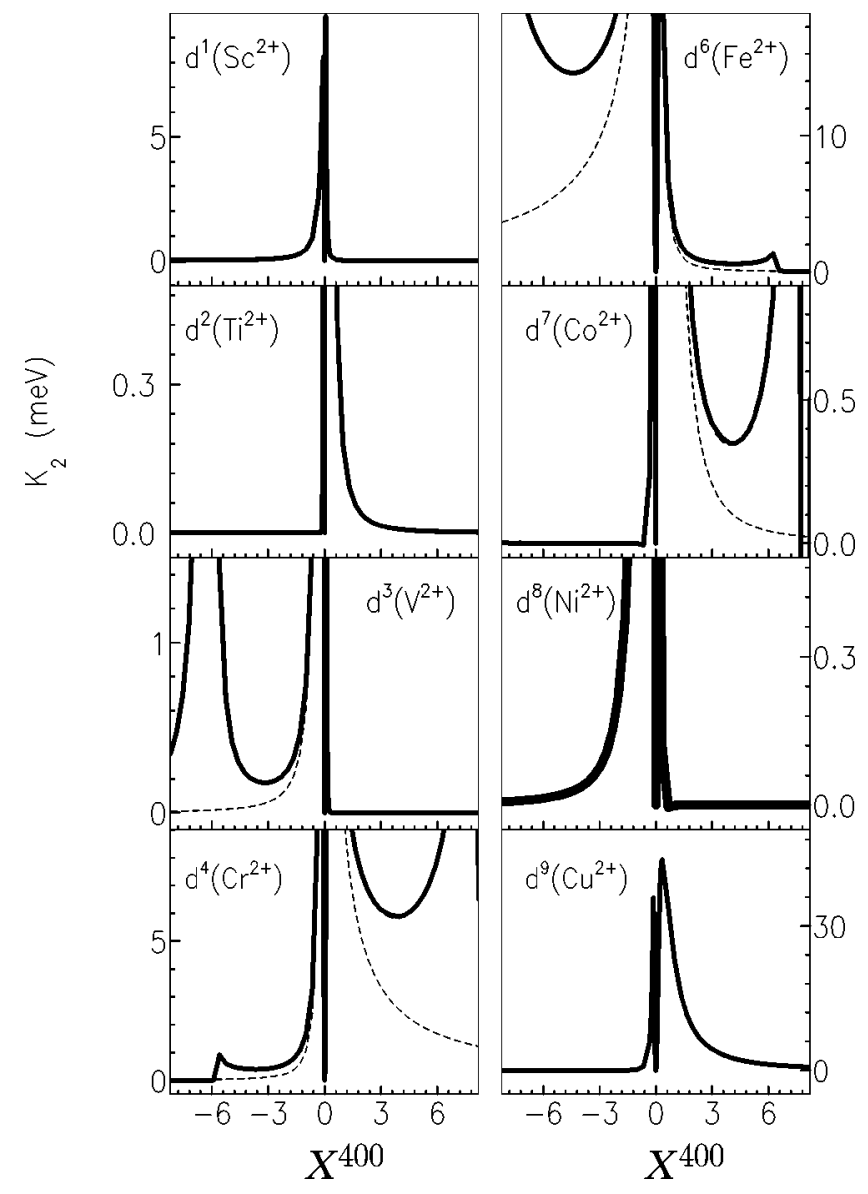

FIG. 5. Calculated cubic single-ion anisotropy constant $K_{2}^{g}$ as a function of the cubic CF parameter $X^{400}$ and a constant exchange field of $\mu_{0} \mu_{B} H_{\mathrm{ex}}=50 \mathrm{meV}$ at $T=0 \mathrm{~K}$. Full lines give the result from the complete CF theory, in which, as described in Sec. II the basis set includes all terms. Dashed lines represent the result of a basis set that include only the Hund's rule $(L, S, J)$ term. These calculations have been carried out within the CF approach of Sec. III, with $A_{4}^{0}=X^{400} / 72 \sqrt{30} \theta_{4}$.

mate an accurate value for the exchange field from the temperature dependence of the (site dependent) magnetic moment. Results for specific materials will be presented in Sec. VI. The range of values of $X^{400}$ for which $K_{1}$ and $K_{2}$ are given corresponds for the $d^{1}$ case to a $t_{2 g}-e_{g}$ level splitting of up to $2.5 \mathrm{eV}$.

The configurations $d^{n}$ and $d^{10-n}$ are related by the fact that configurations with more than five electrons may be treated in the same way by considering holes in a filled shell instead of electrons. As a result, the general shape of the $X^{400}$ dependence of $K_{1}$ and $K_{2}$ is for such related pairs of configurations very similar when comparing curves for opposite crystal fields. Of course, the absolute values can be highly different, as the spin-orbit parameter increases with increasing $n$.

In the limit of a small $\mathrm{CF}$ as compared to spin-orbit coupling, $K_{1}$ is proportional to the product of the asphericity of the charge cloud times the asphericity of the potential. Therefore the sign changes of $K_{1}$ when going from $d^{1}$ to $d^{9}$ correlate with the sign changes of the Stevens factor $\theta_{4}$ (see Sec. III). 


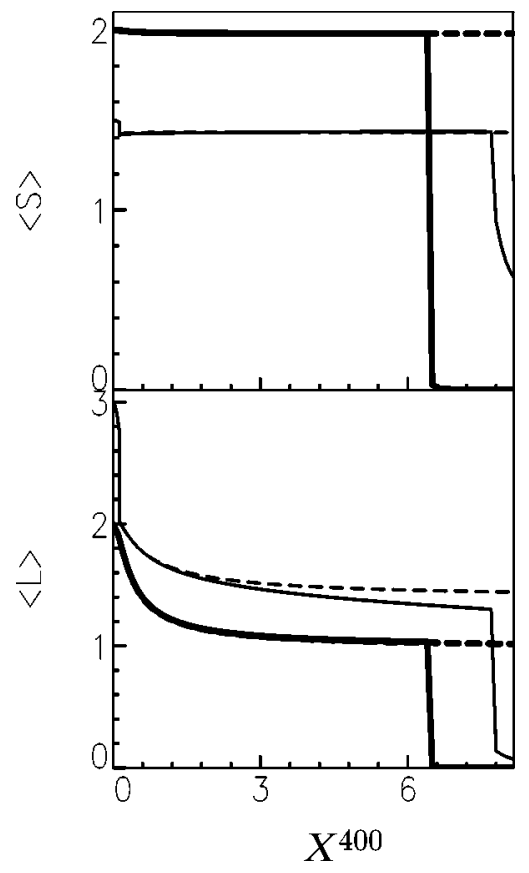

FIG. 6. Expectation value of the spin and orbital moment along the [111] direction as a function of the cubic CF parameter $X^{400}$ at $T=0 \mathrm{~K}$. Full (dashed) lines correspond to the full multiplet structure (Hund's rule term only). The thick (thin) lines correspond to $\mathrm{Fe}^{2+}\left(\mathrm{Co}^{2+}\right)$, with $\mu_{0} \mu_{B} H_{\mathrm{ex}}=50 \mathrm{meV}$ along the [111] direction.

The characteristic maximum in the single-ion MCA constants, for crystal fields of the order of $\lambda$, coincides for both models considered here (see the Appendix). However, for larger values of $X^{400}$ differences are visible, with the exception of the cases $d^{1}$ and $d^{9}$. For these latter configurations there is only one term symbol and therefore both approaches are equivalent. This means that a method based on the restricted Hamiltonian discussed in Sec. III, which only operates on the Hund's rule ground state, is only adequate for very small values of the CF. In a solid one has to deal with crystal fields which are generally much larger. Therefore, the method as based on the restricted Hamiltonian is in most cases of practical interest inadequate.

Large differences in the single-ion MCA constants are found using the restricted Hamiltonian as compared with the full multiplet theory for $d^{3}\left(T_{d}\right), d^{7}\left(O_{h}\right), d^{4}$, and $d^{6}$ [both $\left(T_{d}\right)$ and $\left.\left(O_{h}\right)\right]$. For these configurations a high-spin to lowspin transition occurs above a certain critical CF parameter $X^{400}$. Before the actual transition takes place the ground state becomes heavily mixed under the influence of excited states, which leads to the large differences in the single-ion MCA constants as observed for the two methods considered.

Let us take a closer look at the effect of a high-spin to low-spin transition on the expectation value of the spin and orbital moment for $d^{6}\left(\mathrm{Fe}^{2+}\right)$ (thin lines) and $d^{7}\left(\mathrm{Co}^{2+}\right)$ (thick lines) at $T=0 \mathrm{~K}$ shown in Fig. 6. The spin moment is only slightly influenced by the crystal field until at $X^{400}$ $=6.5(7.8)$ for $d^{6}\left(d^{7}\right)$ a sharp decrease of the spin value takes place. The orbital moment is readily quenched from the free ion value for small values of the crystal field and becomes almost zero after the (sudden) high-spin to low-spin transition. The value for both the spin and orbital moment as obtained on the basis of the restricted Hamiltonian (acting on the Hund's rule ground state) remains finite above $X^{400}$ $=6.5(7.8)$ for $d^{6}\left(d^{7}\right)$, as expected. Before the high-spin to low-spin transition occurs one observes already a relatively large difference between the Hund's rule term orbital moment of $d^{7}$ and the full multiplet theory moment. This latter effect is caused by mixing in of character from excited states before the actual crossing of energy levels occurs.

On the basis of the cubic spin-Hamiltonian of ions whose orbital moment is quenched by the CF one would expect that anisotropy disappears for $S<2 .{ }^{33}$ Figures 4 and 5 indeed show that the anisotropy for ions with $S<2$ is smaller as compared to $S=2$, but it does not vanish for $S<2$. One should note, however, that here the orbital part of the magnetic moment is not fully quenched over the entire parameter range shown and that the theoretical prediction based on the cubic spin-Hamiltonian neglects the influence of mixing in to the ground state of states belonging to multiplets other than the Hund's rule ground state multiplet.

\section{B. Local trigonal symmetry}

Figure 7 shows the global cubic single ion MCA constants $K_{1}^{g}$ and $K_{2}^{g}$ [as defined in Eq. (18)] for a system with ions at sites with local trigonal symmetry around the four equivalent $\langle 111\rangle$ directions at $T=0 \mathrm{~K}$. As discussed above, this is, e.g., the case encountered for the contribution to the anisotropy from cations at octahedral sites in the spinel structure. The figure gives results for all $3 d^{n}(n=1-4,6-9)$ configurations as a function of the local trigonal CF parameter $X^{2 \tilde{1} 0}$, for a constant value of the cubic CF parameter $X^{400}$ $=18 / \sqrt{30}$, which for the case of a $d^{1}$ configuration corresponds to a $t_{2 g}-e_{g}$ level splitting of $1.0 \mathrm{eV}$. A value of $X^{210}$ equal to one (minus one) corresponds to a $a_{1}-e$ splitting of $0.33(0.38) \mathrm{eV}$. The exchange field used, corresponding to $\mu_{0} \mu_{B} H_{\mathrm{ex}}=50 \mathrm{meV}$, is equal to the value used for the cubic case in Sec. V A.

For all configurations, except of course for $d^{1}$ and $d^{9}$, one observes a shift in the characteristic maximum of the single ion MCA constants. As discussed in the Appendix for $d^{1}$ this maximum is caused by interactions within the ground state term $(L, S, J)$. The figure shows that a trigonal $\mathrm{CF}$ can in some cases have a very strong effect on the position of this maximum (e.g., for the $d^{2}$ configuration with $X^{2 \tilde{1} 0}>0$ ), obviously since a crossing of energy levels may also be influenced by mixing in of terms at higher energy. This then changes the value of both the ground state spin and orbital moment and therefore influences the position of the maximum. The figure makes clear that a fit to experimentally observed single-ion MCA constants would in many cases yield two sets of completely different parameters when using the full and restricted Hamiltonian presented here. A clear and very relevant example is the case $3 d^{7}\left(\mathrm{Co}^{2+}\right)$. For a large positive $\mathrm{CF}\left(X^{2 \tilde{1} 0}=1\right)$, the predicted value of $K_{1}\left(K_{2}\right)$ are obtained from the full and the restricted Hamiltonians are 


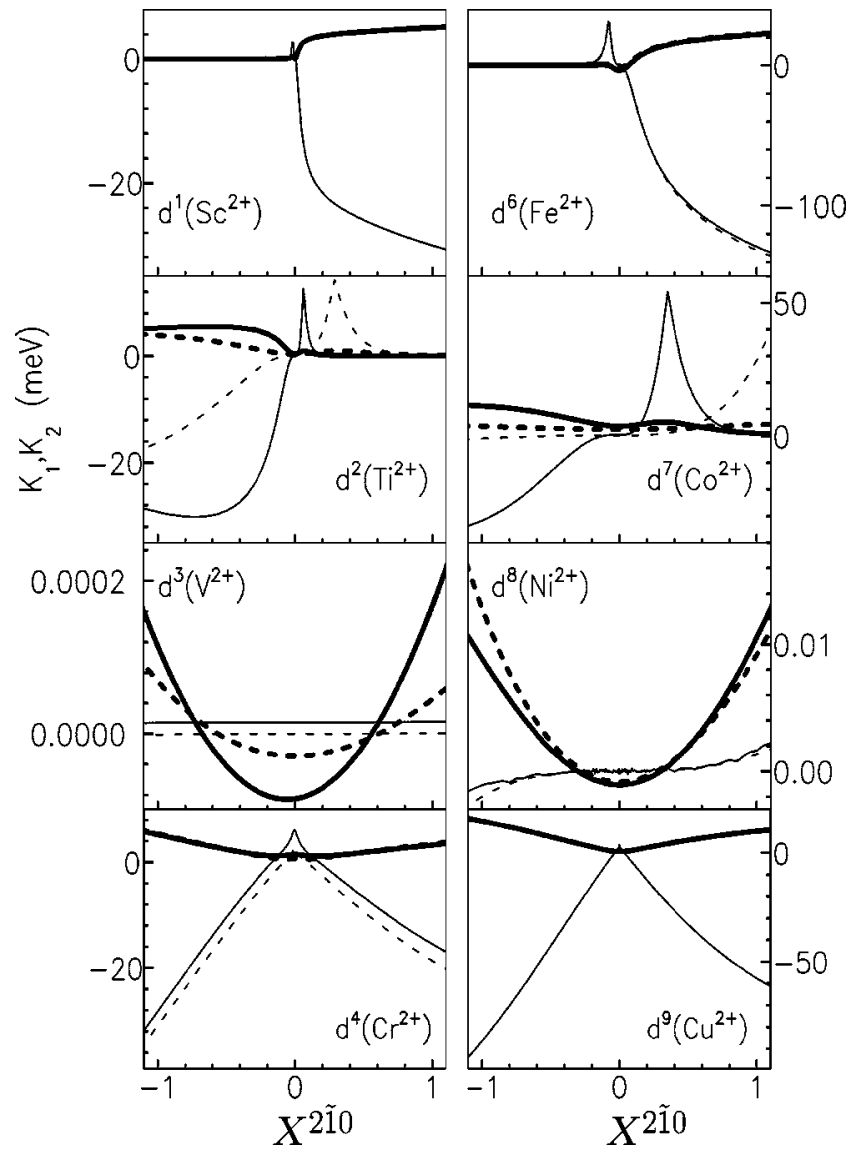

FIG. 7. Calculated cubic single-ion anisotropy constants $K_{1}^{g}$ (thick) and $K_{2}^{g}$ (thin) as a function of the local trigonal parameter $X^{2 \tilde{1} 0}$ on top of a constant octahedral CF splitting of $X^{400}=18 / \sqrt{30}$ $(\Delta=1.0 \mathrm{eV})$, and a constant exchange field of $\mu_{0} \mu_{B} H_{\mathrm{ex}}=50 \mathrm{meV}$ at $T=0 \mathrm{~K}$. Full lines give the result from the complete CF theory, in which, as described in Sec. II the basis set includes all terms. The full lines for $\mathrm{V}^{2+}\left(d^{3}\right)$ are obtained by a spline fit to the calculated data points. Dashed lines represent the result of a basis set that include only the Hund's rule $(L, S, J)$ term. These calculations have been carried out within the CF approach of Sec. III, with $A_{2}^{0}$ $=X^{2 \tilde{1} 0} /-3 \sqrt{70} \theta_{2}$.

different by a factor of 2.9(22.5). For a large negative CF $\left(X^{2 \tilde{10}}=-1\right)$ these factors are $0.5(0.027)$. We return to this case in Sec. VI.

The single ion MCA constants of $d^{n}$ and $d^{10-n}$ are not in the same sense related as is the case for pure cubic symmetry. There is, however, a close resemblance between $d^{n}$ and $d^{5+n}$, since we focus here onto the effect of the $t_{2 g}$ orbitals. Suppose we have two sets of energy levels, which each may be internally splitted by crystal field effects, separated by a large (intra-atomic) exchange field. In such a case a half filled shell behaves as if it is empty, since these filled energy levels of a certain spin kind, say spin up, are isolated from the spin down levels and do not contribute to the single-ion MCA.

\section{Local tetragonal symmetry}

Figure 8 shows the global cubic single-ion MCA constants $K_{1}^{g}$ and $K_{2}^{g}$ for systems with ions at sites with local
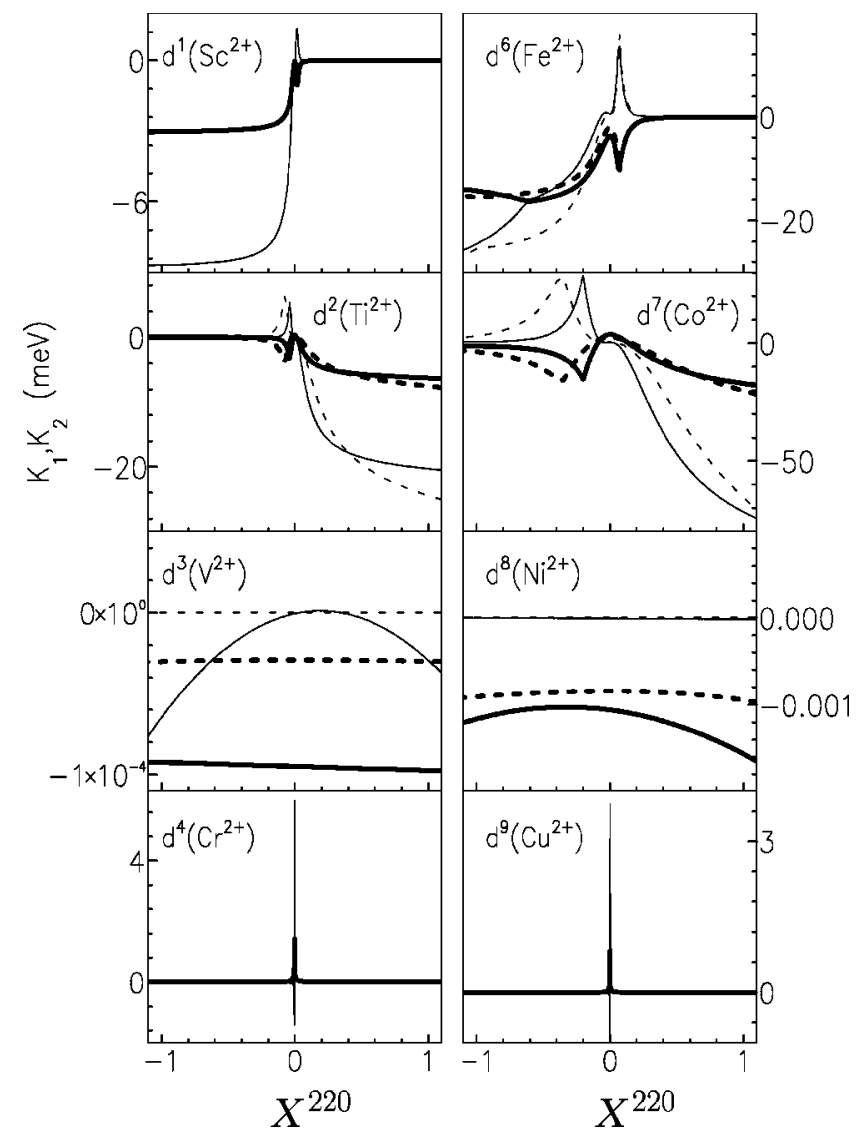

FIG. 8. Calculated cubic single-ion anisotropy constants $K_{1}^{g}$ (thick) and $K_{2}^{g}$ (thin) as a function of the local tetragonal parameter $X^{220}$ on top of a constant octahedral CF splitting of $X^{400}=18 / \sqrt{30}$ $(\Delta=1.0 \mathrm{eV})$, and a constant exchange field of $\mu_{0} \mu_{B} H_{\mathrm{ex}}=50 \mathrm{meV}$ at $T=0 \mathrm{~K}$. Full lines give the result from the complete CF theory, in which, as described in Sec. II the basis set includes all terms. The full lines for $\mathrm{V}^{2+}\left(d^{3}\right)$ and $\mathrm{Ni}^{2+}\left(d^{8}\right)$ are obtained by a spline fit to the calculated data points. Dashed lines represent the result of a basis set that include only the Hund's rule $(L, S, J)$ term. These calculations have been carried out within the CF approach of Sec. III, with $A_{2}^{0}=X^{220} /-3 \sqrt{70} \theta_{2}$.

tetragonal symmetry around three equivalent $\langle 100\rangle$ directions, for all $3 d^{n}(n=1-4,6-9)$ configurations at $T=0 \mathrm{~K}$. Also here we use a fixed CF parameter $X^{400}=18 / \sqrt{30}$ and an exchange field corresponding to $\mu_{0} \mu_{B} H_{\mathrm{ex}}=50 \mathrm{meV}$, as used in Secs. V A and V B. A value of $X^{220}$ equal to one (minus one) corresponds to a $a_{1}-b_{1}\left(e-b_{2}\right)$ splitting of $0.36(0.48) \mathrm{eV}$.

A comparison with the results given in Sec. V B for ions on sites with a local trigonal symmetry reveals that the variation with $-X^{220}$ (Fig. 8) of the global cubic single-ion MCA constants is roughly similar with $+X^{2 \tilde{10}}$ (Fig. 7). A negative value of the tetragonal CF parameter $X^{220}$ creates in a single electron picture a doublet $e$ ground state while a positive parameter $X^{2 \tilde{1} 0}$ in trigonal symmetry does the same. Of course the details in both cases are different, leading to the differences as observed.

\section{RESULTS FOR SPECIFIC MATERIALS}

In this section we discuss the contribution to the MCA of tetrahedrally coordinated $\mathrm{Ni}^{2+}$ and octahedrally coordinated 
$\mathrm{Fe}^{2+}$ and $\mathrm{Co}^{2+}$ in nickel ferrite $\left(\mathrm{NiFe}_{2} \mathrm{O}_{3}\right)$, magnesiummanganese ferrite $\left(\mathrm{Mg}_{x} \mathrm{Mny} \mathrm{Fe}_{3-x-y} \mathrm{O}_{4}\right)$, and lithium-cobalt ferrite $\left(\mathrm{Li}_{0.5-x / 2} \mathrm{Fe}_{2.5-x / 2} \mathrm{Co}_{x} \mathrm{O}_{4}\right)$, respectively. For these systems experimental data, taken at or close to $4.2 \mathrm{~K}$, are available from the literature on the basis of which the single-ion MCA due to the ions of interest can be separated out. In the case of $\mathrm{Ni}^{2+}$ in nickel ferrite the MCA as calculated using the full and the restricted Hamiltonian is more or less the same. We have nevertheless selected this example, to clarify how the observed MCA can be explained. Previous theoretical work did not result in an explanation. In the case of $\mathrm{Fe}^{2+}$ on octahedral sites the use of the full Hamiltonian, instead of the restricted Hamiltonian, is found to lead to moderate changes of the calculated value of $K_{1}$ and $K_{2}$, but for $\mathrm{Co}^{2+}$ on octahedral sites the theory based on the restricted Hamiltonian is qualitatively and quantitatively incorrect. Some attention will be paid to the role of the value of the spin-orbit parameter $\left(\zeta_{3 d}\right)$ used. The comparison with experimental results suggest that a reduction of $\zeta_{3 d}$, as compared to the free ion values ${ }^{21}$ is required.

First we start with $\mathrm{Ni}^{2+}$ in nickel ferrite. In its thermodynamically stable state this compound has the inverse spinel structure in which one half of the octahedrally coordinated (B) sites is occupied by $\mathrm{Ni}^{2+}$ ions, while the other half of the octahedral sites and all tetrahedral sites are occupied by $\mathrm{Fe}^{3+}$. The anisotropy due to octahedral $\mathrm{Ni}^{2+}$ with (Fig. 7) or without a trigonal distortion (Fig. 4) is certainly no more negative than $-1 \times 10^{-6} \mathrm{eV}$ (it can in fact be positive up to $10 \times 10^{-6} \mathrm{eV}$ in the range shown for $X^{2 \tilde{1} 0}$ ). The more negative experimentally observed anisotropy constant $K_{1}=-5.3$ $\times 10^{-6} \mathrm{eV} /$ atom for nickel ferrite has therefore been attributed mainly to $\mathrm{Fe}^{3+} .{ }^{33}$ The single-ion MCA for this ion is small due to the fact that the orbital moment for $\mathrm{Fe}^{3+}\left(d^{5}\right)$ is almost fully quenched.

Experimental results have shown that the MCA is much higher when $\mathrm{Ni}^{2+}$ is located at the tetrahedral $(A)$ sites. This can be accomplished by rapidly quenching from its sintering temperature to room temperature. The experimental single ion MCA constant of $\mathrm{Ni}^{2+}$ at the tetrahedral sites is $K_{1}$ $=2.7 \mathrm{meV}$ at $T=4.2 \mathrm{~K}^{37}$ The random distribution of twoand three-valent ions at the $B$ sites brings about the existence of low-symmetry crystal fields at the $A$ sites of the spinel structure. Therefore the first theoretical interpretations were based on tetragonal ${ }^{9}$ and trigonal distortions ${ }^{37}$ of the tetrahedral site. As far as we know the most recent discussion on this issue has been presented by Novak. ${ }^{38}$ In his evaluation of the MCA he used perturbation theory on the ${ }^{3} T_{1}$ multiplet. He showed that within this theoretical framework a pure (undistorted) tetrahedral $\mathrm{CF}$ cannot explain the experimental MCA, since the exchange field needed would be unrealistically high. Secondly, he concluded that the low symmetry crystal fields due to tetrahedral or trigonal distortions, as mentioned above, cannot explain the experimental results observed. In contrast, we have found that a satisfactory explanation of the experimental results can be obtained, already in the absence of such distortions, on the basis of the more complete theories that we consider here. In Fig. 9 we show our results obtained for $K_{1}$ and $K_{2}$ of $\mathrm{Ni}^{2+}$ at a tetrahedral

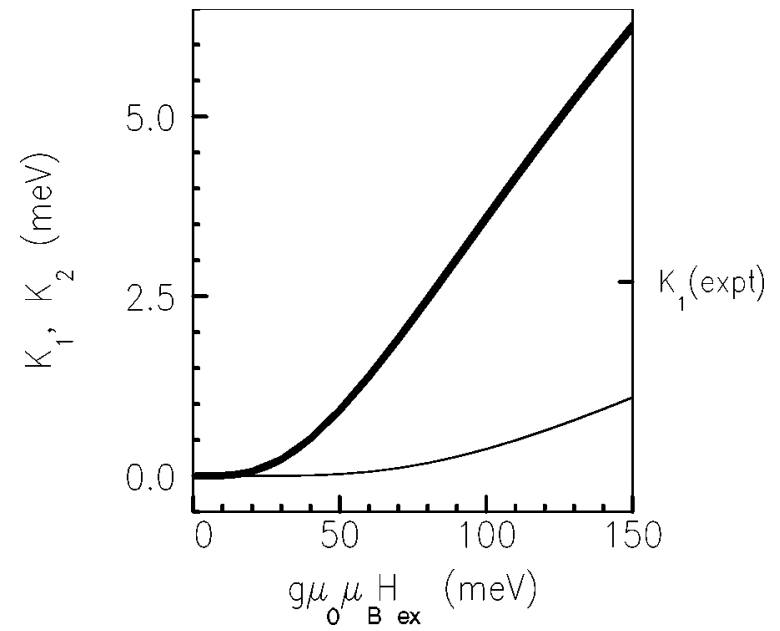

FIG. 9. Calculated cubic single-ion anisotropy constants $K_{1}^{g}$ (thick) and $K_{2}^{g}$ (thin) for $\mathrm{Ni}^{2+}$ as a function of the exchange field at $T=0 \mathrm{~K}$. We use a tetrahedral CF splitting of $X^{400}=-9 / \sqrt{30}(\Delta=$ $-0.5 \mathrm{eV})$. These calculations have been carried out within the $\mathrm{CF}$ approach as described in Sec. III and the complete CF theory, in which, as described in Sec. II, the basis set includes all terms. Both methods give within $\approx 1 \times 10^{-6} \mathrm{eV}$ the same result.

symmetry site as a function of the exchange field. The methods based on the theory as described in Secs. II and III give within $\approx 1 \times 10^{-6} \mathrm{eV}$ the same result, indicating that mixing in of character of excited states outside ${ }^{3} F$ is unimportant for this example. We use a tetrahedral crystal field parameter of $\Delta=-0.5 \mathrm{eV}\left(X^{400}=-9 / \sqrt{30}\right)$ as obtained from Ref. 39. A value of the exchange, viz., $g \mu_{0} \mu_{B} H_{\mathrm{ex}}=81 \mathrm{meV}$ would explain the experimental value of $K_{1}=2.7 \mathrm{meV}$. This value is just slightly above the upper limit as given by Novak. ${ }^{38}$ Unfortunately, an accurate experimental value of the exchange field is unknown. Finally, we would like to remark that a possible trigonal distortion does not significantly change the results, while a static Jahn-Teller-like tetragonal distortion, as suggested in Ref. 9 would give the wrong sign.

The second example considered is the single ion contribution of $\mathrm{Fe}^{2+}$ at octahedral sites in spinel-type ferrites. Experimental studies of the contribution of $\mathrm{Fe}^{2+}$ to $K_{1}$ and $K_{2}$, at liquid helium temperatures, have made use of changes in the $\mathrm{Fe}^{2+}$ concentration as a consequence of the substitution of $\mathrm{Ti}^{4+}$ for $\mathrm{Fe}^{3+}$ (Ref. 40) or of changes taking place in conjunction with changes of the concentration of manganese ferrites. ${ }^{41}$ In the former case the substitution of the $\mathrm{Ti}^{4+}$ ion, which has a high valence and a smaller ionic radius than $\mathrm{Fe}^{2+}$, induces a polarization and strain in the lattice. In the latter case a gradual transition of the structure from the normal to the inverse spinel structure takes place. These side effects can considerably influence the magnitude of the measured anisotropy contribution of the $\mathrm{Fe}^{2+}$ ion. Therefore we consider the results of these studies on the $\mathrm{Fe}^{2+}$ contributions to the $K_{1}$ and $K_{2}$ as less reliable.

Such side effects are, to our opinion, much less disturbing in the case of a study of the single-ion MCA for $\mathrm{Fe}^{2+}$ ions at the octahedral (B) sites in the ferrite $\mathrm{Mg}_{x} \mathrm{Mn}_{y} \mathrm{Fe}_{3-x-y} \mathrm{O}_{4}$, with $0 \leqslant x \leqslant 0.63 \mathrm{eV}^{42}$ resulting in $K_{1}=2.7 \times 10^{-5} \mathrm{eV}$ per $\mathrm{Fe}^{2+}$, for $T \rightarrow 0 \mathrm{~K}$. Unfortunately, $K_{2}$ was not given. In fact, 


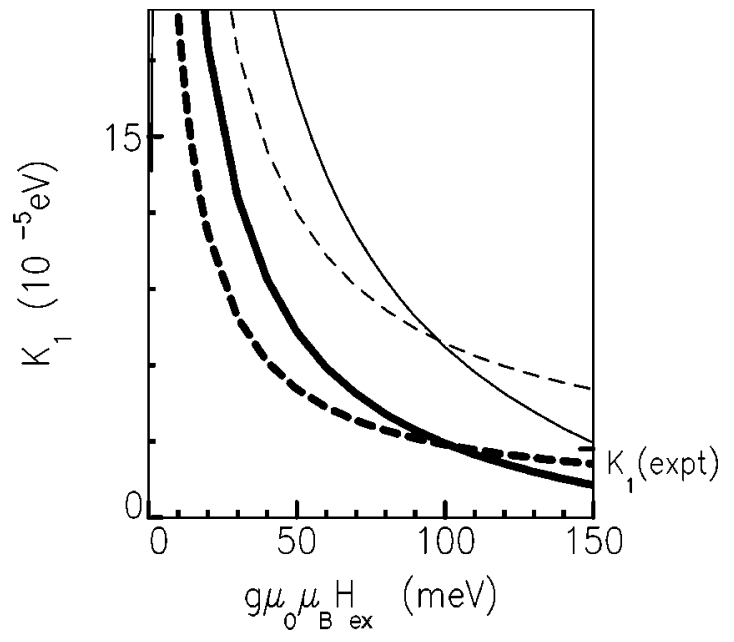

FIG. 10. Calculated cubic single-ion anisotropy constants $K_{1}^{g}$ for $\mathrm{Fe}^{2+}$ as a function of the exchange field at $T=0 \mathrm{~K}$. We use an octahedral CF parameter $X^{400}=9 \sqrt{3 / 10}(\Delta=1.5 \mathrm{eV})$ and a trigonal CF parameter $X^{2 \tilde{1} 0}=-0.55\left(X_{t}=-0.19 \mathrm{eV}\right)$, for both the HF spinorbit parameter $\lambda(100 \%$; thin) and a reduced value of $\lambda(80 \%$; thick). Full lines give the result from the complete CF theory, in which, as described in Sec. II the basis set includes all terms. Dashed lines represent the result of a basis set that include only the Hund's rule $(L, S, J)$ term. These calculations have been carried out within the CF approach of Sec. III, with $A_{4}^{0}=X^{400} / 72 \sqrt{30} \theta_{4}$ and $A_{2}^{0}=X^{2 \tilde{1} 0} /-3 \sqrt{70} \theta_{2}$.

the octahedral sites at which the $\mathrm{Fe}^{2+}$ ions reside are trigonally distorted. As the cubic and trigonal CF for this class of compounds is not known experimentally, we have carried out a calculation using parameters as obtained for related systems. The temperature dependent quadrupole splitting of the hyperfine spectrum of $\mathrm{Fe}^{2+}$ ions in $\mathrm{GeFe}_{2} \mathrm{O}_{4}$ has been interpreted in terms of a cubic (trigonal) CF splitting of $\Delta$ $=1.8 \mathrm{eV}\left(X_{t}=-0.2 \mathrm{eV}\right) .{ }^{43}$ Here $X_{t}$ is the splitting which arises (within a one electron picture) between states of $e$ and $a_{1}$, symmetry. For $\mathrm{Fe}_{3} \mathrm{O}_{4}$ the cubic $\mathrm{CF}$ splitting has been obtained from x-ray absorption spectroscopy (XAS) (Ref. 44 ) and Kerr spectra, ${ }^{45}$ yielding on average $\Delta=1.5 \mathrm{eV}$. Although these results have been obtained for different ferrites than considered here they give a good first indication concerning the size and sign of the crystal field parameters. In Fig. 10 we present $K_{1}$ calculated for $\mathrm{Fe}^{2+}$ at octahedral sites using $X^{400}=4.93(\Delta=1.5 \mathrm{eV})$ with a trigonal $\mathrm{CF}$ parameter $X^{2 \tilde{10}}=-0.55\left(X_{t}=-0.2 \mathrm{eV}\right)$ as a function of the exchange field. A value of the exchange field corresponding to $g \mu_{0} \mu_{B} H_{\mathrm{ex}}=100 \mathrm{meV}$ would explain the experimental value of $K_{1}=2.7 \times 10^{-5} \mathrm{eV}$. ${ }^{42}$ The calculations have been performed using a spin-orbit parameter $\zeta_{3 d}$ that is $80 \%$ of the spin-orbit parameter that was obtained for the free ion. ${ }^{20}$ Without this reduction of the spin-orbit parameter it is not possible to explain the experimental value of $K_{1}$, since it would require unrealistically high exchange fields (see Fig. 10). We return to this issue in the next section and remark here that a similar reduction of $\zeta_{3 d}$ for the case of $\mathrm{Ni}^{2+}$ (discussed above), would only marginally change the predicted value of $K_{1}$ and $K_{2}$ at the value of $H_{\text {ex }}$ given $(10 \%$

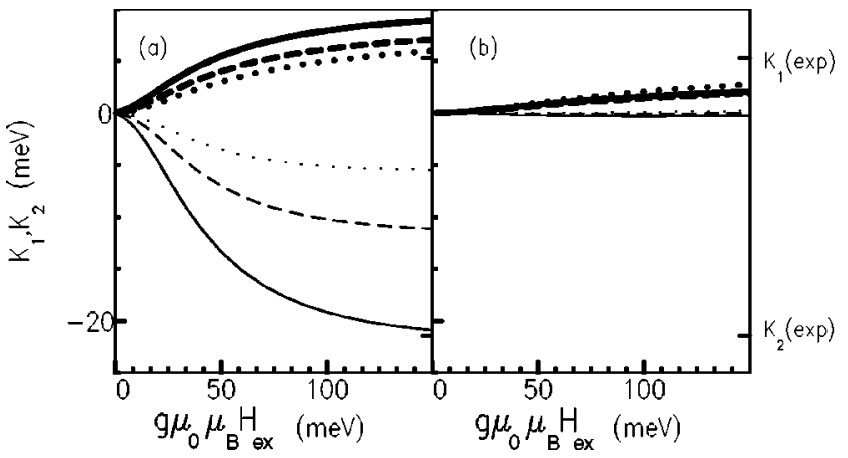

FIG. 11. Calculated cubic single-ion anisotropy constants $K_{1}^{g}$ (thick) and $K_{2}^{g}$ (thin) for $\mathrm{Co}^{2+}$ as a function of the exchange field at $T=0 \mathrm{~K}$. We used a reduced HF value $(80 \%)$ of the spin-orbit parameter $\lambda$ for three different combinations of the CF parameters: (i) $X^{400}=9 \sqrt{3 / 10}(\Delta=1.5 \mathrm{eV})$ and $X^{2 \tilde{1} 0}=-0.35\left(X_{t}=-0.12 \mathrm{eV}\right)$ (full lines), (ii) $X^{400}=9 \sqrt{3 / 10}(\Delta=1.5 \mathrm{eV})$ and $X^{2 \tilde{10}}=-0.25\left(X_{t}=\right.$ $-0.09 \mathrm{eV})$ (dashed lines), (iii) $X^{400}=18 / \sqrt{30}(\Delta=1.0 \mathrm{eV})$ and $X^{2 \tilde{1} 0}=-0.25\left(X_{t}=-0.09 \mathrm{eV}\right)$ (dotted lines). (a) These calculations have been carried out within the full CF Hamiltonian for which, as described in Sec. II, the basis set includes all terms. (b) These calculations have been carried out with a basis set that include only the Hund's rule $(L, S, J)$ term, with $A_{4}^{0}=X^{400} / 72 \sqrt{30} \theta_{4}$ and $A_{2}^{0}$ $=X^{2 \tilde{1} 0} /-3 \sqrt{70} \theta_{2}$.

reduction of $K_{1}, \approx 1 \%$ reduction of $K_{2}$ ). Although there are differences between the two models used as described in Secs. II and III, for the case of $\mathrm{Fe}^{2+}$ ions discussed above they more or less accidentally coincide in the parameter range of interest (see Fig. 10). Finally we want to mention that the values obtained for $K_{2}$ are positive and of the same order of magnitude as $K_{1}$.

The third and final example concerns the experimentally observed single-ion MCA constants $K_{1}=5.3 \mathrm{meV}$ and $K_{2}$ $=-21.4 \mathrm{meV}$ for $\mathrm{Co}^{2+}$ at the octahedral $(B)$ sites of ordered $\mathrm{Li}_{0.5-x / 2} \mathrm{Fe}_{2.5-x / 2} \mathrm{Co}_{x} \mathrm{O}_{4}$, with $0 \leqslant x \leqslant 0.005$ at $T=4.2 \mathrm{~K}^{46}$ The $\mathrm{Co}^{2+}$ ions occupy octahedral sites and experience a trigonal field produced by the averaged charge distribution at the $B$ sites. The first theoretical interpretations, based on the spin Hamiltonian approach, failed to describe the results obtained at low temperature. ${ }^{47,48}$ In the Introduction we already mentioned the results by Baltzer. ${ }^{9}$ Using a similar method as presented here in Sec. II, he obtained for the single ion MCA constants $K_{1}=5.9 \mathrm{meV}$ and $K_{2}=-18.6 \mathrm{meV}$, with $g \mu_{0} \mu_{B} H_{\text {ex }}=70 \mathrm{meV}$ and a trigonal field of $10 \%$ of the cubic field strength, which is very close to the experimental values. In Fig. 11 [(a) full Hamiltonian Sec. II, (b) restricted Hamiltonian Sec. III] we present $K_{1}$ and $K_{2}$ calculated for $\mathrm{Co}^{2+}$ at octahedral sites with a trigonal distortion as a function of the exchange field. The crystal fields used are indicated in the figure caption. It is clear that a theory based on the restricted Hamiltonian fails completely, while the full theory leads to a much better, though not perfect, agreement with experiment. A parameter set of $g \mu_{0} \mu_{B} H_{\mathrm{ex}}=80 \mathrm{meV}, X^{400}=4.93(\Delta$ $=1.5 \mathrm{eV}), X^{2 \tilde{10}}=-0.35\left(X_{t}=-0.12 \mathrm{eV}\right)$ and $\zeta_{3 d}$ reduced to $80 \%$ of the free ion value ${ }^{20}$ would give a reasonably good explanation of the experimental data. 


\section{DISCUSSION}

Analyses of the single ion contribution to the MCA on the basis of the full Hamiltonian presented in Sec. II have been shown to provide for some systems a large improvement to analyses based on the restricted Hamiltonian (Sec. III), but they still suffer from uncertainties with respect to the parameter values to be used and from several weaknesses of the model itself. In this section we discuss these issues.

The first uncertainty concerns the value of the CF parameters and (in the case of partly disordered systems) their distribution. In Sec. VI we have referred to one system for which the parameters have been derived from the analysis of Mössbauer spectra and to one system for which XAS was used. It would be very interesting to use the latter modern technique, or other modern atom resolved techniques such as $\mathrm{x}$-ray magnetic linear dichroism or x-ray magnetic circular dichroism $^{15}$ to get more accurate ion specific information about the $\mathrm{CF}$ parameters. In addition, results of $a b$ initio band structure theory within the local spin density approximation can be used to obtain the CF parameters. In the absence of independent experimental or theoretical knowledge about the $\mathrm{CF}$ parameters, they can only be considered as fit parameters.

A similar uncertainty concerns the value of the exchange field. The values obtained for the corresponding energy $g \mu_{0} \mu_{B} H_{\text {ex }}$ were for all examples discussed in Sec. VI relatively high, in the range $80-100 \mathrm{meV}$, when considering that such values would correspond in a mean field model for a ferromagnetic system with only one crystallographic distinct site to $T_{c} \approx 1700 \mathrm{~K}$ (although when correlation beyond mean field theory would be taken into account, $T_{c}$ would be lower ${ }^{49}$ ). These high values could be the result of failures of the theoretical approach used, but could also be partly due to our poor quantitative understanding of the exchange interaction in these compounds. It is known that the molecular field theory overestimates the exchange field at the $A$ sites of ferrites. ${ }^{50}$ In a recent attempt to compare this theory with the theory of superexchange one even had to assume the exchange field at the A sites to be zero. ${ }^{51}$ Moreover, the influence of charge transfer was neglected, which could be especially important for the second halve of the first row TM oxides. $^{52}$

The model which leads to the full CF Hamiltonian presented in Sec. II is known to posses several weaknesses. Maybe the most important weakness is that it neglects the overlap of magnetic ions' wave functions with those of neighboring ions. ${ }^{21,24}$ Hybridization will therefore change the character of the ground state, which for more covalent materials cannot be neglected. For oxidic materials configuration interaction can be described by a mixing of TM $d$-orbital character with $O p$ character. This results in the introduction of $d^{N+1} L$ character in the ground state, where $L$ denotes a hole in the oxygen band and an extra electron in the TM $d$ states. For ions at cubic sites this results effectively in an additional ("ligand field") splitting of the $t_{2 g}$ and $e_{g}$ state (within a one-electron picture). This effect is usually in fact considered to be the largest contribution to the experimentally observed splitting. As it can be included in the CF pa- rameters, it does not degrade the usability of the model. However, a second consequence of the effect of charge transfer [mixing in of $O(2 p)$ character in $d$ states] is an effective reduction of the spin-orbit parameter. We have already mentioned in Sec. V that a reduction of the single-ion spin-orbit parameter (to approximately $80 \%$ of the original value) is required to obtain a reasonable explanation of the experimental anisotropy due to $\mathrm{Fe}^{2+}$ and $\mathrm{Co}^{2+}$ on octahedral sites in spinel-type ferrites. More work is required in order to make clear to what extent such an effective reduction can be applied to many classes of oxides, or that it is highly system dependent.

\section{CONCLUSIONS}

We have shown that the calculation of the single-ion contribution to the MCA, on the basis of a theory that takes the full multiplet structure into account, may lead to significantly different values of the single-ion MCA constants as compared to the ones obtained on the basis of standard $\mathrm{CF}$ theory, which only takes the Hund's rule ground term into account. This has been demonstrated for atoms with $d^{n}$ ( $n$ $=1-4,6-9$ ) configurations, at sites with a local cubic, tetragonal or trigonal symmetry. This demonstrates that mixing in of wave function character from states within excited multiplets is sometimes significant.

Three example are given which were already extensively discussed in the literature both experimentally and theoretically using various models, i.e., the single-ion MCA contribution of $\mathrm{Fe}^{2+}, \mathrm{Co}^{2+}$, and $\mathrm{Ni}^{2+}$ in spinel-type ferrites. It is demonstrated that for reasonable parameters one can get a fairly good explanation of the experimental values. In the case of $\mathrm{Ni}^{2+}$ it is shown that the influence of mixing in of character of excited states is unimportant. The contribution of $\mathrm{Fe}^{2+}$ represents an intermediate case in the sense that the anisotropy parameter $K_{1}$ as derived from both models presented here accidentally coincide in the parameter range of interest. The final example of $\mathrm{Co}^{2+}$ can only be understood by including the excited states. In order to increase the predictive power of the theory presented more accurate information about the $\mathrm{CF}$ and exchange field parameters in these systems is required. It would be very interesting to use detailed information about the element specific crystal field parameters obtained from a modern technique such as XAS to narrow down the number of free parameters.

\section{ACKNOWLEDGMENTS}

We would like to thank Dr. B. G. Searle for using his computer program. The research of D.A. has been made possible by the Netherlands Technology Foundation (STW).

\section{APPENDIX}

In this appendix we discuss the characteristic maximum of the single-ion MCA constants for small values, of the order of the spin-orbit parameter $\lambda$, of the CF parameters. In order to demonstrate the physical origin of this peaking we use the example of a model Hamiltonian for $d^{1}$ ions (such as 
$\mathrm{Sc}^{2+}$ ) at a lattice site with cubic point symmetry. In this case $\mathcal{H}_{d d}$, the Hamiltonian that represents $d$ - $d$ Coulomb interactions, is zero, since there is only one electron involved. We further simplify this example by taking the limit of $\mu_{0} \mu_{B} H_{\mathrm{ex}} \rightarrow \infty$, so that we end up with a convenient $5 \times 5$ matrix, consisting of matrix elements $\left\langle\phi_{i}\left|\mathcal{H}_{\mathrm{CF}}\right| \phi_{j}\right\rangle$ of the $\mathrm{CF}$ Hamiltonian $\mathcal{H}_{\mathrm{CF}}$ between the real spherical harmonic orbital $\left|\phi_{i}, \downarrow\right\rangle$ indicated above and to the left of the matrix. For the case of the exchange field along the [001] direction the matrix is given by

$\left.\begin{array}{c|ccccc}d_{x y}^{\downarrow} & d_{y z}^{\downarrow} & d_{z^{2}}^{\downarrow} & d_{x z}^{\downarrow} & d_{x^{2}-y^{2}}^{\downarrow} \\ d_{x y}^{\downarrow} & -4 D q & 0 & 0 & 0 & -i \lambda \\ d_{y z}^{\downarrow} & 0 & -4 D q & 0 & \frac{-i \lambda}{2} & 0 \\ d_{z^{2}}^{\downarrow} & 0 & 0 & 6 D q & 0 & 0 \\ d_{x z}^{\downarrow} & \frac{i \lambda}{2} & 0 & -4 D q & 0 \\ d_{x^{2}-y^{2}}^{\downarrow} & 0 & 0 & 0 & 6 D q\end{array}\right)$.

The resulting energies are

$$
E_{1}=6 D q,
$$

$$
\begin{gathered}
E_{2,3}=-4 D q \pm \frac{\lambda}{2}, \\
E_{4,5}=D q \pm \sqrt{25(D q)^{2}+\lambda^{2}} .
\end{gathered}
$$

It is now easy to see that a crossing occurs, i.e., a change of ground state, as a function of the CF parameter $\Delta$ at a fixed value of $\lambda$ at $\Delta=\frac{3}{2} \lambda$. As a result, the dependence of the MCA, which is the dependence of the ground state energy of the direction on the magnetization (i.e., of the exchange field), changes suddenly at this point. Physically, there is a transition from eigenstates of $L_{z}$ (for $\lambda \gg \Delta$ ) to eigenstates of crystal field (for $\lambda \ll \Delta$ ). Eigenstates of $L_{z}$ correspond to the tendency of the orbital angular momentum to be opposite to the spin direction. Indeed, the results from a full calculation for $\mathrm{Sc}^{2+}$ in Fig. 4 (with a finite value of $H_{\mathrm{ex}}$, discussed in Sec. V), show such a sudden change in the dependence of the cubic anisotropy parameter $K_{1}$ at a value of the crystal field parameter $X^{400}=49 \times 10^{-3}$, which corresponds to $\Delta$ $=\sqrt{30} / 18 \times 49=14.9 \mathrm{meV}$, whereas $\frac{3}{2} \lambda\left(\mathrm{Sc}^{2+}\right)=16.5 \mathrm{meV}$. This effect is present in all $3 d^{n}$ configurations and originates within one $(L, S)$ term. While the exact position of the maximum is a function of all interactions, it is clear that a sudden change of the ground state, for a certain range of directions of the exchange field, as a function of the $\mathrm{CF}$ is responsible for the effect.
*Present address: Philips Research Laboratories, Prof. Holstlaan 4, 5656 AA Eindhoven, The Netherlands. The FORTRAN program used for the calculations as described in Sec. III is available from the first author on request.

${ }^{1}$ J. Smit and H. P. J. Wijn, in Ferrites, Philips Technical Library (Wiley, New York, 1959).

${ }^{2}$ R. von Helmolt, J. Wecker, B. Holzapfel, L. Schultz, and K. Samwer, Phys. Rev. Lett. 71, 2331 (1993).

${ }^{3}$ M. J. Carey and A. E. Berkowitz, Appl. Phys. Lett. 60, 3060 (1992)

${ }^{4}$ P. J. van der Zaag, A. R. Ball, L. F. Feiner, R. M. Wolf, and P. A. A. van der Heijden, J. Appl. Phys. 79, 5103 (1996).

${ }^{5}$ M. I. Darby and E. D. Isaac, IEEE Trans. Magn. 10, 259 (1974), and references therein.

${ }^{6}$ Magnetic Oxides, edited by D. J. Craik (Wiley, New York, 1975), Vol. 1, Chaps. 3 and 5.

${ }^{7}$ C. Ruduwicz, Magn. Reson. Rev. 13, 1 (1987)

${ }^{8}$ H. B. Callen and E. Callen, J. Phys. Chem. Solids 27, 1271 (1966).

${ }^{9}$ P. K. Baltzer, J. Phys. Soc. Jpn. 17, 192 (1962); Ph.D. thesis, Rutgers University, 1963.

${ }^{10}$ M. D. Kuz'min and J. M. D. Coey, Phys. Rev. B 50, 12533 (1994).

${ }^{11}$ N. W. Ashcroft and N. D. Mermin, in Solid State Physics (W. B. Saunders, Philadelphia, 1976).

${ }^{12}$ Y. Tanabe and S. Sugano, J. Phys. Soc. Jpn. 9, 766 (1954).

${ }^{13}$ R. D. Cowan, J. Opt. Soc. Am. 58, 808 (1968); R. D. Cowan, in The Theory of Atomic Structure and Spectra (University of California Press, Berkeley, 1981).

${ }^{14}$ P. H. Butler, in Point Group Symmetry, Applications, Methods and Tables (Plenum, New York, 1981).

${ }^{15}$ B. T. Thole, G. van der Laan, and G. A. Sawatzky, Phys. Rev. Lett. 55, 2086 (1985).

${ }^{16}$ G. van der Laan, J. Electron Spectrosc. Relat. Phenom. 86, 41 (1997).

${ }^{17}$ F. M. F. de Groot, J. C. Fuggle, B. T. Thole, and G. A. Sawatzky, Phys. Rev. B 42, 5459 (1990).

${ }^{18}$ D. Alders, L. H. Tjeng, G. A. Sawatzky, F. C. Voogt, T. Hibma, J. Vogel, M. Sacchi, S. Iacobucci, and C. T. Chen, Phys. Rev. B 57, 11623 (1998).

${ }^{19}$ G. van der Laan, B. T. Thole, and G. A. Sawatzky, Phys. Rev. B 37, 6587 (1988).

${ }^{20}$ E. U. Condon and G. H. Shortley, in The Theory of Atomic Spectra (Cambridge University Press, Cambridge, 1935).

${ }^{21}$ J. Owen and J. H. M. Thornley, Rep. Prog. Phys. 29, 675 (1966).

${ }^{22}$ B. G. Wybourne, in Spectroscopic Properties of Rare Earths (Interscience, New York, 1965).

${ }^{23}$ G. Racah, Phys. Rev. 62, 438 (1942).

${ }^{24}$ M. T. Hutchings, Solid State Phys. 16, 227 (1964).

${ }^{25}$ K. W. H. Stevens, Proc. Phys. Soc., London, Sec. A 65, 209 (1952).

${ }^{26}$ C. Ruduwicz, J. Phys. C 18, 1415 (1985).

${ }^{27}$ H. A. Buckmaster, Can. J. Phys. 40, 1670 (1962).

${ }^{28}$ D. Smith and J. H. M. Thornley, Proc. Phys. Soc. London 89, 779 (1966).

${ }^{29}$ R. J. Birgeneau, Can. J. Phys. 45, 3761 (1967).

${ }^{30}$ R. Fieschi and F. G. Fumi, Nuovo Cimento 10, 865 (1953).

${ }^{31}$ J. J. M. Franse and G. de Vries, Physica (Amsterdam) 39, 477 (1968).

${ }^{32}$ R. F. Pearson, in Experimental Magnetism, edited by G. M. 
Kalvins and R. S. Tebble (Wiley, New York, 1979).

${ }^{33}$ K. Yosida and M. Tachiki, Prog. Theor. Phys. 17, 331 (1957).

${ }^{34}$ W. P. Wolf, Phys. Rev. 108, 1152 (1957).

${ }^{35}$ J. C. Slonczewski, J. Appl. Phys. 32, 253 (1961).

${ }^{36}$ R. Gersdorf, Phys. Rev. Lett. 40, 344 (1978).

${ }^{37}$ A. J. Pointon, J. M. Robertson, and G. A. Wetton, J. Phys. (France) 32, 850 (1971).

${ }^{38}$ P. Novak, Czech. J. Phys., Sect. B 22, 1135 (1972).

${ }^{39}$ D. Reinen, Struct. Bonding (Berlin) 7, 114 (1970).

${ }^{40}$ J. Smit, F. K. Lotgering, and R. P. van Stapele, J. Phys. Soc. Jpn. 17, 2064 (1962).

${ }^{41}$ A. Broese van Groenou and R. F. Pearson, J. Phys. Chem. Solids 28, 1027 (1967).

${ }^{42}$ R. Gerber and G. Elbinger, J. Phys. C 3, 1363 (1970).

${ }^{43}$ M. Eibschütz, U. Ganiel, and S. Shtrikman, Phys. Rev. 151, 245
(1966).

${ }^{44}$ P. Kuiper, B. G. Searle, L.-C. Duda, R. M. Wolf, and P. J. van der Zaag, J. Electron Spectrosc. Relat. Phenom. 86, 107 (1997).

${ }^{45}$ W. F. Fontijn, P. J. van der Zaag, M. A. C. Devillers, V. A. M. Brabers, and R. Metselaar, Phys. Rev. B 56, 5432 (1997).

${ }^{46}$ V. N. Seleznev, I. K. Pukhov, A. I. Dronin, and V. A. Shapovalov, Sov. Phys. J. 12, 683 (1970).

${ }^{47}$ J. C. Slonczewski, Phys. Rev. 110, 1341 (1958).

${ }^{48}$ M. Tachiki, Prog. Theor. Phys. 23, 1055 (1960).

${ }^{49}$ J. Callaway, Quantum Theory of the Solid States, 2nd ed. (Academic, New York, 1991), Chap. 4.

${ }^{50}$ G. F. Dionne, J. Appl. Phys. 85, 4627 (1999).

${ }^{51}$ P. G. Bercoff and H. R. Bertorello, J. Magn. Magn. Mater. 169, 314 (1997).

${ }^{52}$ J. Zaanen and G. A. Sawatzky, Can. J. Phys. 65, 1262 (1987). 